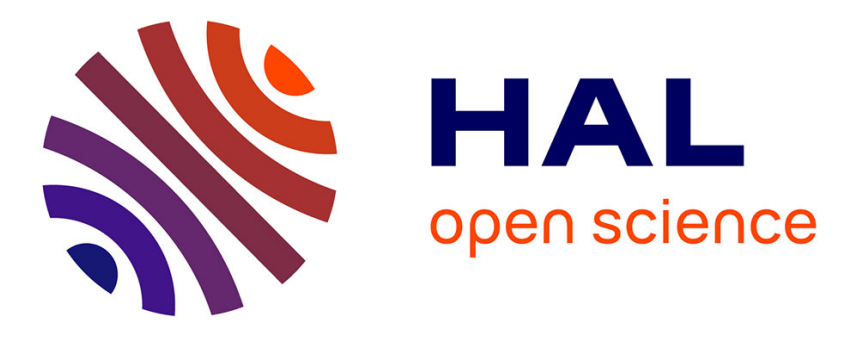

\title{
The CPHD filter with target spawning
}

Daniel S. Bryant, Emmanuel Delande, Steven Gehly, Jeremie Houssineau, Daniel Clark, Brandon Jones

\section{To cite this version:}

Daniel S. Bryant, Emmanuel Delande, Steven Gehly, Jeremie Houssineau, Daniel Clark, et al.. The CPHD filter with target spawning. IEEE Transactions on Signal Processing, 2017, 65 (5), pp.13241338. 10.1109/TSP.2016.2597126. hal-01730369

\section{HAL Id: hal-01730369 https://hal.science/hal-01730369}

Submitted on 13 Mar 2018

HAL is a multi-disciplinary open access archive for the deposit and dissemination of scientific research documents, whether they are published or not. The documents may come from teaching and research institutions in France or abroad, or from public or private research centers.
L'archive ouverte pluridisciplinaire HAL, est destinée au dépôt et à la diffusion de documents scientifiques de niveau recherche, publiés ou non, émanant des établissements d'enseignement et de recherche français ou étrangers, des laboratoires publics ou privés. 


\title{
The CPHD Filter With Target Spawning
}

\author{
Daniel S. Bryant, Emmanuel D. Delande, Steven Gehly, Jérémie Houssineau, Daniel E. Clark, and Brandon A. Jones
}

\begin{abstract}
In its classical form, the cardinalized probability hypothesis density (CPHD) filter does not model the appearance of new targets through spawning, yet there are applications for which spawning models more appropriately account for newborn objects when compared to spontaneous birth models. In this paper, we propose a principled derivation of the CPHD filter prediction step including spontaneous birth and spawning. A Gaussian Mixture implementation of the CPHD filter with spawning is then presented, illustrated with three applicable spawning models on a simulated scenario involving two parent targets spawning a total of five objects.
\end{abstract}

Index Terms-Multi-object filtering, Cardinalized probability hypothesis density (CPHD) filter, point processes, random finite sets, Bayesian estimation, target tracking, target spawning.

\section{INTRODUCTION}

$\mathbf{T}$ HE goal of the multi-object estimation problem is to jointly estimate - usually in the presence of clutter, data association uncertainty, and missed detections - the time-varying number and individual states of targets evolving in a surveillance scene. Commonly known detection and tracking algorithms for the multi-object problem include joint probabilistic data association (JPDA) [1] and multiple hypothesis tracking (MHT) [2]. Relatively new is the multi-object filtering framework known as finite set statistics (FISST) [3], [4], based on a representation of the target population as a random finite set (RFS), a specific case of the more general concept of point process.

Within the FISST framework, the multi-target Bayes filter proposes an optimal solution to the multi-object estimation problem. Several approximations of the multi-target Bayes filter have been proposed to circumvent challenges associated with its complexity, including the probability hypothesis density (PHD) [5]

Manuscript received October 23, 2015; revised February 18, 2016, April 15 , 2016, and July 7, 2016; accepted July 11, 2016. Date of publication August 8,2016 ; date of current version January 6,2017 . The associate editor coordinating the review of this manuscript and approving it for publication was Prof François Desbouvries. The work of D. S. Bryant was supported by the Science, Mathematics \& Research for Transformation Scholarship-for-Service Program. The work of E. D. Delande and D. E. Clark was supported by the Engineering and Physical Sciences Research Council Platform under Grant EP/J015180/1 and the MOD University Defence Research Centre on Signal Processing Phase 2 (EP/K014227/1).

D. S. Bryant and S. Gehly are with the Department of Aerospace Engineering Sciences, University of Colorado Boulder, Boulder, CO 80309 USA (e-mail: daniel.bryant@colorado.edu; steven.gehly@ colorado.edu).

E. D. Delande, J. Houssineau, and D. E. Clark are with the School of Engineering and Physical Sciences, Heriot-Watt University, Edinburgh EH14 4AS, U.K. (e-mail: E.D.Delande@hw.ac.uk; j.houssineau@hw.ac.uk; d.e.clark@hw.ac.uk).

B. A. Jones is with the Department of Aerospace Engineering and Engineering Mechanics, University of Texas at Austin, Austin, TX 78712 USA (e-mail: brandon.jones@utexas.edu).

Color versions of one or more of the figures in this paper are available online at http://ieeexplore.ieee.org.

Digital Object Identifier 10.1109/TSP.2016.2597126 and the CPHD [6] filters. The PHD filter propagates the firstorder factorial moment density, or intensity, of the multi-target RFS, representing the whole population of targets within the surveillance scene [5]. While computationally inexpensive, the PHD filter exhibits a high variability in the estimated target number [4]. The CPHD filter [6] addresses this issue by estimating the cardinality distribution of the multi-target RFS in addition to its intensity. Unlike for the PHD filter, the initial presentation of the CPHD filter does not include a model for target spawning in the time prediction step. Target spawning refers to instances where a parent target generates one or more daughter targets and where the daughter(s) usually remain(s) in close proximity to the parent for some amount of time following their appearance, e.g., a fighter jet that launches a missile.

Though the CPHD filter's model for birth targets has the potential to address spawning targets [4], there may be cases where specific spawning models are more applicable. Consider for example the case of tracking resident space objects (RSOs), natural and artificial Earth orbiting satellites consisting of active spacecraft, decommissioned payloads, and debris. In this context, spawning events include the deployment of CubeSats from a launch vehicle [7], [8] and fragmentation events caused by the unintentional [9] or intentional [10] collision of objects. Without spawning, the best option may be the use of diffuse birth regions, however, the volume of space to be filled requires a potentially intractable number of birth regions [11]. To improve the CPHD filter's performance for spaceobject tracking, previous research has presented a measurementbased birth model that leverages an astrodynamics approach to track initialization for RSOs [12]. While such an approach may be effective for tracking spawned RSOs, a multi-target filter that more accurately describes the physical processes that produce new RSOs through a specific spawning model is expected to provide better accuracy and faster confirmation of new objects.

In this paper, we extend the usual CPHD filter [5] with an arbitrary spawning term and present our results through the partial Bell polynomials [13] to facilitate practical implementation of the extended CPHD time prediction equation. The incorporation of spawning models in the context of CPHD filtering has previously been explored in [14], relying on an intuitive construction of the filtering equations for Bernoulli or Poisson spawning models via traditional Bayesian statistics. A technical report by the same authors additionally presents an overview of a more principled derivation of the predicted cardinality [15], whose results match some of our own once their presentation has been rearranged. While an approximation is made in [14] to circumvent evaluation of a complex integral for the implementation of a CPHD filter with a Poisson spawning model, we present in this paper the CPHD time prediction equation for an arbitrary spawning process, and then for three specific models 
(Poisson, Bernoulli, zero-inflated Poisson), without necessity for additional approximations.

Another work deals with CPHD spawning [16] that cites and forms comparisons with an earlier version of the current article [17]. The most significant difference between presented results is that a conclusive analytical expression for the predicted cardinality distribution that includes arbitrary birth and spawning is not provided in [16], i.e., quantities remain requiring further derivation by the reader, whereas the current article presents conclusive, analytical and tractable expressions in Section III-B. Additionally, while derivations in this article include the application of Faà di Bruno's formula to probability generating functionals (p.g.fl.s) describing point processes, the approach in [16] is more comparable to that of the technical report [15] in that both apply the formula to probability generating functions (p.g.fs) describing probability mass functions.

The structure of this paper is as follows. Section II presents the relevant background on point processes and functional differentiation, followed by key definitions and properties pertinent to our results. Section III provides a detailed construction of the CPHD filter with target spawning, considering several models of spawning processes. Section IV demonstrates the proposed concepts through simulation example, and closing remarks are given in Section V. A pseudo-code for the CPHD cardinality prediction with spawning is given in Section VI. The proofs of the results in Section III are given in the Appendix.

\section{BACKGROUND}

In this section, we introduce the necessary background on point processes (Section II-A), on p.g.fl.s (Section II-B), on functional differentiation (Section II-C), and on a few properties from the application of differentiation in the context of point processes (Section II-D). Section II-E then provides a brief description of the general multi-target Bayes filter [3], and the principled approximation leading to the construction of the original CPHD filter [6].

\section{A. Point Processes}

A point process on some space $\mathbf{X}$ is a random variable whose number of elements and element states, belonging to $\mathbf{X}$, are random. In the context of multi-target tracking the population of targets is represented by a point process $\Phi$, on a single-target state space $\mathbf{X} \subseteq \mathbb{R}^{d}$, whose elements describe individual target states. A realization of $\Phi$ is a vector of points $\varphi=\left(x_{1}, \ldots, x_{N}\right)$ depicting a specific multi-target configuration, where $x_{i} \in \mathbf{X}$ describes the $d$-component state of an individual target (position, velocity, etc.).

A point process $\Phi$ is characterized by its probability distribution $P_{\Phi}$ on the measurable space $\left(\mathcal{X}, \mathbf{B}_{\mathcal{X}}\right)$, where $\mathcal{X}=$ $\bigcup_{n \geq 0} \mathbf{X}^{n}$ is the point process state space, i.e., the space of all the finite vectors of points in $\mathbf{X}$, and $\mathbf{B}_{\mathcal{X}}$ is the Borel $\sigma$-algebra on $\mathcal{X}$ [18]. The probability distribution of a point process is defined as a symmetric function, so that the order of points in a realization is irrelevant for statistical purposes - for example, realizations $\left(x_{1}, x_{2}\right)$ and $\left(x_{2}, x_{1}\right)$ are equally probable. In addition, if the probability distribution is such that the realizations are vectors of points that are pairwise distinct almost surely, then the point process is called simple. For the rest of the paper, all the point processes are assumed simple. ${ }^{1}$

The probability distribution $P_{\Phi}$ is characterized by its projection measures $P_{\Phi}^{(n)}$, for any $n \geq 0$. The $n^{\text {th }}$-order projection measure $P_{\Phi}^{(n)}$, for any $n \geq 1$, is defined on the Borel $\sigma$-algebra of $\mathbf{X}^{n}$ and gives the probability for the point process to be composed of $n$ points, and the probability distribution of these points. By extension, $P_{\Phi}^{(0)}$ is the probability for the point process to be empty. For any $n \geq 0, J_{\Phi}^{(n)}$ denotes the $n^{\text {th }}$-order Janossy measure [20, p. 124], and is defined as

$$
\begin{aligned}
J_{\Phi}^{(n)}\left(B_{1} \times \ldots \times B_{n}\right) & =\sum_{\sigma(n)} P_{\Phi}^{(n)}\left(B_{\sigma_{1}} \times \ldots \times B_{\sigma_{n}}\right) \\
& =n ! P_{\Phi}^{(n)}\left(B_{1} \times \ldots \times B_{n}\right),
\end{aligned}
$$

where $B_{i}$ is in $\mathbf{B}_{\mathbf{X}}$, the Borel $\sigma$-algebra of $\mathbf{X}, 1 \leq i \leq n$, and where $\sigma(n)$ denotes the set of all permutations $\left(\sigma_{1}, \ldots, \sigma_{n}\right)$ of $(1, \ldots, n)$.

The probability density $p_{\Phi}$ (respectively (resp.) the $n^{\text {th }}$-order projection density $p_{\Phi}^{(n)}$, the $n^{\text {th }}$-order Janossy density $j_{\Phi}^{(n)}$ ) is the Radon-Nikodym derivative of the probability distribution $P_{\Phi}$ (resp. the $n^{\text {th }}$-order projection measure $P_{\Phi}^{(n)}$, the $n^{\text {th }}$-order Janossy measure $J_{\Phi}^{(n)}$ ) with respect to (w.r.t.) some reference measure. All these quantities provide equivalent ways to describe the point process $\Phi$. However, a measure-theoretical formulation provides a more general framework that is required to construct certain statistical properties on point processes that can be exploited for practical applications; a recent example is given in [21] for the construction of the regional statistics. For the sake of generality, the rest of the paper thus uses a measurebased description.

Assuming that $f$ is a non-negative measurable function on $\mathcal{X}$, then the integral of $f$ w.r.t. to the measure $P_{\Phi}$ can be written in the following ways:

$$
\begin{aligned}
P_{\Phi}(f)= & \int_{\mathcal{X}} f(\varphi) P_{\Phi}(\mathrm{d} \varphi) \\
= & \int_{\mathcal{X}} f(\varphi) p_{\Phi}(\varphi) \mathrm{d} \varphi \\
= & \sum_{n \geq 0} \int_{\mathbf{X}^{n}} f\left(x_{1}, \ldots, x_{n}\right) P_{\Phi}^{(n)}\left(\mathrm{d}\left(x_{1}, \ldots, x_{n}\right)\right) \\
= & \sum_{n \geq 0} \int_{\mathbf{X}^{n}} f\left(x_{1}, \ldots, x_{n}\right) p_{\Phi}^{(n)}\left(x_{1}, \ldots, x_{n}\right) \\
& \times \mathrm{d} x_{1} \ldots \mathrm{d} x_{n} \\
= & \sum_{n \geq 0} \frac{1}{n !} \int_{\mathbf{X}^{n}} f\left(x_{1}, \ldots, x_{n}\right) J_{\Phi}^{(n)}\left(\mathrm{d}\left(x_{1}, \ldots, x_{n}\right)\right) \\
= & \sum_{n \geq 0} \frac{1}{n !} \int_{\mathbf{X}^{n}} f\left(x_{1}, \ldots, x_{n}\right) j_{\Phi}^{(n)}\left(x_{1}, \ldots, x_{n}\right) \\
& \times \mathrm{d} x_{1} \ldots \mathrm{d} x_{n} .
\end{aligned}
$$

\footnotetext{
${ }^{1}$ An alternative construction of simple point processes as random objects whose realizations are sets of points $\varphi=\left\{x_{1}, \ldots, x_{N}\right\}$, in which the elements are per construction unordered, is also available in the literature [6], [19]. In this context, a point process is called a RFS.
} 
Throughout this article the exploitation of the Janossy measures will be preferred, for they are convenient tools in the context of functional differentiation (see Section II-C). For the sake of simplicity, domains of integration will be omitted when they refer to the full target state space $\mathbf{X}$.

The Janossy measures can also be used directly to exploit meaningful information on the point process $\Phi$. For example, central to this article is the extraction of the cardinality distribution $\rho_{\Phi}$ of the point process, that describes the number of elements in the realizations of $\Phi$ (see Section III):

Example 1 (Cardinality distribution): Consider the function $f_{n}$ defined as

$$
f_{n}(\varphi)= \begin{cases}1, & |\varphi|=n \\ 0, & \text { otherwise }\end{cases}
$$

where $|\varphi|$ denotes the size of the vector $\varphi$. The integral of $f_{n}$ w.r.t. to $P_{\Phi}$ yields the probability $\rho_{\Phi}(n)$ that a realization $\varphi$ of the point process $\Phi$ has size $n$ and we have, using Eq. (2) (see $[22$, p. 28$])$ :

$$
\begin{aligned}
\rho_{\Phi}(n) & =P_{\Phi}\left(f_{n}\right) \\
& =\int_{\mathbf{X}^{n}} P_{\Phi}^{(n)}\left(\mathrm{d}\left(x_{1}, \ldots, x_{n}\right)\right) \\
& =\frac{1}{n !} \int_{\mathbf{X}^{n}} J_{\Phi}^{(n)}\left(\mathrm{d}\left(x_{1}, \ldots, x_{n}\right)\right) .
\end{aligned}
$$

The function $\rho_{\Phi}$ is called the cardinality distribution of the point process $\Phi$. Note that the $n^{\text {th }}$-order projection measure $P_{\Phi}^{(n)}$ (resp. the $n^{\text {th }}$-order Janossy measure $J_{\Phi}^{(n)}$ ) is not a probability measure, in the general case, for its integral over $\mathbf{X}^{n}$ yields $\rho_{\Phi}(n)$ (resp. $\left.n ! \rho_{\Phi}(n)\right)$.

\section{B. Probability Generating Functionals}

The p.g.fl. provides a useful characterization for point process theory [23] and is defined as follows.

Definition 1 (Probability generating functional [20]): The probability generating functional $G_{\Phi}$ of a point process $\Phi$ on $\mathbf{X}$ can be written for any test function $h \in \mathcal{U}(\mathbf{X})$ as $^{2,3}$

$$
\begin{aligned}
G_{\Phi}(h)= & \int_{\mathcal{X}}\left[\prod_{x \in \varphi} h(x)\right] P_{\Phi}(\mathrm{d} \varphi) \\
= & J_{\Phi}^{(0)}+\sum_{n \geq 1} \frac{1}{n !} \int_{\mathbf{X}^{n}} h\left(x_{1}\right) \ldots h\left(x_{n}\right) \\
& \times J_{\Phi}^{(n)}\left(\mathrm{d}\left(x_{1}, \ldots, x_{n}\right)\right) .
\end{aligned}
$$

The p.g.fl. $G_{\Phi}$ fully characterizes the point process $\Phi$, and is a very convenient tool for the extraction of statistical information on $\Phi$ through functional differentiation (see Section II-C). From Eq. (5) we can immediately write

$$
\begin{aligned}
& G_{\Phi}(0)=J_{\Phi}^{(0)}\left(=P_{\Phi}^{(0)}\right), \\
& G_{\Phi}(1)=1 .
\end{aligned}
$$

${ }^{2} \mathcal{U}(\mathbf{X})$ is the space of bounded measurable functions $u$ on $\mathbf{X}$ satisfying $\|u\|_{\infty} \leq 1$.

${ }^{3}$ When $\varphi \in X^{n}, n \geq 0$, is a vector of distinct elements on some space $X$, the abuse of notation " $x \in \varphi$ " is used for " $x \in \chi(\varphi)$ ", where $\chi$ is the function associating a vector of distinct elements to the set composed of the same elements.
Operations on point processes (e.g., superposition of two populations) can be translated into operations on their corresponding p.g.fl.s. In the context of multi-target tracking, p.g.fl.s provide a convenient description of the compound population (targets or measurements) resulting from an operation on elementary populations.

The superposition operation for point processes describes the union of two populations $\Phi_{1}, \Phi_{2}$ into a compound population $\Phi_{1} \cup \Phi_{2}$, during which the information about the origin population of each individual is lost.

Proposition 1 (Superposition of independent processes [23]): Let $\Phi_{1}$ and $\Phi_{2}$ be two independent point processes defined on the same space, with respective p.g.fl.s $G_{\Phi_{1}}$ and $G_{\Phi_{2}}$. The p.g.fl. of the superposition process $\Phi_{1} \cup \Phi_{2}$ is given by the product

$$
G_{\Phi_{1} \cup \Phi_{2}}(h)=G_{\Phi_{1}}(h) G_{\Phi_{2}}(h) .
$$

The Galton-Watson recursion for point processes [23], [24] describes the evolution of each individual $x$ from a parent population $\Phi_{\mathrm{p}}$ into a population of daughter individuals, independently of the other parent individuals but following a common evolution model described by a process $\Phi_{\mathrm{e}}$. The resulting daughter population $\Phi_{\mathrm{d}}$ is then the superposition of all the populations of daughter individuals.

Proposition 2 (The Galton-Watson recursion [24]): Let $G_{\Phi_{\mathrm{p}}}$ be the p.g.fl. of a parent process $\Phi_{\mathrm{p}}$ on $\mathbf{X}$, and let $G_{\Phi_{\mathrm{e}}}(\cdot \mid x)$ be the conditional p.g.fl. of an evolution process $\Phi_{\mathrm{e}}$, defined for every $x \in \mathbf{X}$. The p.g.fl. of the daughter process $\Phi_{\mathrm{d}}$ is given by the composition

$$
G_{\Phi_{\mathrm{d}}}(h)=G_{\Phi_{\mathrm{p}}}\left(G_{\Phi_{\mathrm{e}}}(h \mid \cdot)\right) .
$$

\section{Functional Differentiation}

To make use of functionals in the derivations presented in Section III, we require the notion of differentials on functional spaces. We adopt a restricted form of the Gâteaux differential, known as the chain differential [25], so that a general chain rule can be determined [26], [27]. Following this, we describe the general higher-order chain rule.

Definition 2 (Chain differential [25]): Under the conditions detailed in [25], the function $F$ on some set $H$ has a chain differential $\delta F(h ; \eta)$ at $h \in H$ in the direction $\eta$ if, for any sequence $\eta_{n} \rightarrow \eta \in H$, and any sequence of real numbers $\theta_{n} \rightarrow 0$, it holds that

$$
\delta F(h ; \eta)=\lim _{n \rightarrow \infty} \frac{1}{\theta_{n}}\left(F\left(h+\theta_{n} \eta_{n}\right)-F(h)\right) .
$$

The $n^{\text {th }}$-order chain differential can be defined recursively as

$$
\delta^{n} F\left(h ; \eta_{1}, \ldots, \eta_{n}\right)=\delta\left(\delta^{n-1} F\left(h ; \eta_{1}, \ldots, \eta_{n-1}\right) ; \eta_{n}\right) .
$$

In the context of this paper, as it may be hinted from Definition 1, the chain differential will be applied to probability generating functionals on the single-target state space $\mathbf{X}$, which are functions on the space of test functions $\mathcal{U}(\mathbf{X})$.

Applying $n^{\text {th }}$-order chain differentials on composite functions can be an extremely laborious process since it involves determining the result for each choice of function and proving the result by induction. For ordinary derivatives, the general higher-order chain rule is normally attributed to Faà di Bruno [28]. The following result generalizes Faà di Bruno's formula 
to chain differentials and allows for a systematic derivation of composite functions (see [26], [29] for examples of earlier exploitation in the context of Bayesian estimation).

Proposition 3 (General higher-order chain rule, from [27], [30]): Under the differentiability and continuity conditions detailed in [30], the $n^{\text {th }}$-order variation of composition $F \circ G$ in the sequence of directions $\left(\eta_{i}\right)_{i=1}^{n}$ at point $h$ is given by

$$
\begin{aligned}
\delta^{n} & (F \circ G)\left(h ;\left(\eta_{i}\right)_{i=1}^{n}\right) \\
& =\sum_{\pi \in \Pi_{n}} \delta^{|\pi|} F\left(G(h) ;\left(\delta^{|\omega|} G\left(h ;\left(\eta_{i}\right)_{i \in \omega}\right)\right)_{\omega \in \pi}\right),
\end{aligned}
$$

where $\Pi_{n}=\Pi(\{1, \ldots, n\})$ represents the set of partitions of the index set $\{1, \ldots, n\}$, and $|\pi|$ denotes the cardinality of the set $\pi$.

Example 2 (General higher-order chain rule):

$$
\begin{aligned}
\delta^{2}(F \circ G)\left(h ; \eta_{1}, \eta_{2}\right)= & \underbrace{\delta^{2} F\left(G(h) ; \delta G\left(h ; \eta_{1}\right), \delta G\left(h ; \eta_{2}\right)\right)}_{\pi=\{\{1\},\{2\}\}} \\
& +\underbrace{\delta F\left(G(h) ; \delta^{2} G\left(h ; \eta_{1}, \eta_{2}\right)\right)}_{\pi=\{\{1,2\}\}} .
\end{aligned}
$$

Applying $n^{\text {th }}$-order chain differentials on a product of functions follows a more straightforward approach, similar to Leibniz' rule for ordinary derivatives.

Proposition 4 (Leibniz' rule, from [30]): Under the differentiability conditions detailed in [30], the $n^{\mathrm{th}}$-order variation of the product $F \cdot G$ in the sequence of directions $\left(\eta_{i}\right)_{i=1}^{n}$ at point $h$ is given by

$$
\begin{aligned}
\delta^{n} & (F \cdot G)\left(h ;\left(\eta_{i}\right)_{i=1}^{n}\right) \\
& =\sum_{\pi \subseteq\{1, \ldots, n\}} \delta^{|\pi|} F\left(h ;\left(\eta_{i}\right)_{i \in \pi}\right) \delta^{n-|\pi|} G\left(h ;\left(\eta_{i}\right)_{i \in \pi^{c}}\right),
\end{aligned}
$$

where $\pi^{c}=\{1, \ldots, n\} \backslash \pi$ denotes the complement of $\pi$ in $\{1, \ldots, n\}$.

Example 3 (Leibniz' rule):

$$
\begin{aligned}
\delta^{2} & (F \cdot G)\left(h ; \eta_{1}, \eta_{2}\right) \\
& =\underbrace{\delta^{2} F\left(h ; \eta_{1}, \eta_{2}\right) G(h)}_{\pi=\{1,2\}}+\underbrace{\delta F\left(h ; \eta_{1}\right) \delta G\left(h ; \eta_{2}\right)}_{\pi=\{1\}} \\
& +\underbrace{\delta F\left(h ; \eta_{2}\right) \delta G\left(h ; \eta_{1}\right)}_{\pi=\{2\}}+\underbrace{F(h) \delta G\left(h ; \eta_{1}, \eta_{2}\right)}_{\pi=\{\emptyset\}} .
\end{aligned}
$$

\section{Probability Generating Functionals and Differentiation}

Key properties of a point process can be recovered from the functional differentiation of its p.g.fl. Taking the $k^{\text {th }}$-order variation of $G_{\Phi}(h)$ in the directions $\eta_{1}, \ldots, \eta_{k}$, we have (see, for example [31, p. 21]),

$$
\begin{aligned}
\delta^{k} G_{\Phi}\left(h ; \eta_{1}, \ldots,\right. & \left.\eta_{k}\right)=\sum_{n \geq k} \frac{1}{(n-k) !} \int_{\mathbf{X}^{n}} \prod_{i=1}^{k} \eta_{i}\left(x_{i}\right) \\
& \times \prod_{i=k+1}^{n} h\left(x_{i}\right) J_{\Phi}^{(n)}\left(\mathrm{d}\left(x_{1}, \ldots, x_{n}\right)\right) .
\end{aligned}
$$

It is then useful to consider the cases when we set $h=1$ or $h=0$, i.e.,

$$
\begin{aligned}
\delta^{k} & G_{\Phi}\left(0 ; \eta_{1}, \ldots, \eta_{k}\right) \\
& =\int_{\mathbf{X}^{k}} \eta_{1}\left(x_{1}\right) \ldots \eta_{k}\left(x_{k}\right) J_{\Phi}^{(k)}\left(\mathrm{d}\left(x_{1}, \ldots, x_{k}\right)\right), \\
& \delta^{k} G_{\Phi}\left(1 ; \eta_{1}, \ldots, \eta_{k}\right) \\
& =\int_{\mathbf{X}^{k}} \eta_{1}\left(x_{1}\right) \ldots \eta_{k}\left(x_{k}\right) M_{\Phi}^{(k)}\left(\mathrm{d}\left(x_{1}, \ldots, x_{k}\right)\right),
\end{aligned}
$$

where $M_{\Phi}^{(k)}$ is the $k^{\text {th }}$-order factorial moment measure, defined as in [18, p. 111].

Assuming that one wishes to evaluate the Janossy and factorial moment measures in some measurable subsets $B_{i} \in \mathbf{B}_{\mathbf{X}}$, $1 \leq i \leq k$, then they can be recovered from Eqs. (17) and (18) by setting the directions to be indicator functions ${ }^{4} \eta_{i}=1_{B_{i}}$, $1 \leq i \leq k$, so that

$$
\begin{aligned}
& \left.\delta^{k} G_{\Phi}\left(h ; 1_{B_{1}}, \ldots, 1_{B_{k}}\right)\right|_{h=0}=J_{\Phi}^{(k)}\left(B_{1} \times \ldots \times B_{k}\right), \\
& \left.\delta^{k} G_{\Phi}\left(h ; 1_{B_{1}}, \ldots, 1_{B_{k}}\right)\right|_{h=1}=M_{\Phi}^{(k)}\left(B_{1} \times \ldots \times B_{k}\right) .
\end{aligned}
$$

The propagation of the first-order factorial moment measure $M_{\Phi}^{(1)}$ - also called the intensity measure $\mu_{\Phi}$ - of the multi-target point process $\Phi$, in a Bayesian context, is a key component of the construction of both the PHD filter [5] and the CPHD filter [6]. The density of the intensity measure is called the Probability Hypothesis Density [5].

\section{E. Multiobject Filtering and the CPHD Filter}

The multi-target Bayes filter [3] is the natural extension of the usual single-target Bayesian paradigm to the multi-target case, within the FISST framework. The multi-target Bayes recursion at time step $k$ consists of the time prediction and data update steps given as follows:

$$
\begin{aligned}
P_{k \mid k-1}\left(\mathrm{~d} \varphi \mid Z_{1: k-1}\right) & =\int_{\mathcal{X}} f_{k \mid k-1}(\varphi \mid \bar{\varphi}) P_{k-1}\left(\mathrm{~d} \bar{\varphi} \mid Z_{1: k-1}\right) \\
P_{k}\left(\mathrm{~d} \varphi \mid Z_{1: k}\right) & =\frac{g_{k}\left(Z_{k} \mid \varphi\right) P_{k \mid k-1}\left(\mathrm{~d} \varphi \mid Z_{1: k-1}\right)}{\int_{\mathcal{X}} g_{k}\left(Z_{k} \mid \bar{\varphi}\right) P_{k \mid k-1}\left(\mathrm{~d} \bar{\varphi} \mid Z_{1: k-1}\right)},
\end{aligned}
$$

where $P_{k \mid k-1}$ (resp. $P_{k}$ ) is the probability distribution of the predicted multi-target process $\Phi_{k \mid k-1}$ (resp. the posterior multitarget process $\left.\Phi_{k}\right), Z_{i}, 1 \leq i \leq k$, is the set of measurements collected at time step $i, Z_{1: i}$ denotes the sequence $Z_{1}, \ldots, Z_{i}$, $f_{k \mid k-1}$ is the multi-target transition kernel, and $g_{k}$ is the multitarget likelihood function. The multi-target transition kernel $f_{k \mid k-1}$ describes the time evolution of the population of targets since time step $k-1$ and encapsulates the underlying models of

\footnotetext{
${ }^{4}$ For a measurable subset $B \in \mathbf{B}_{\mathbf{X}}$, the indicator function $1_{B}$ is defined as the function on $\mathbf{X}$ such that $1_{B}(x)=1$ if $x \in B, 1_{B}(x)=0$ otherwise.
} 
target birth, motion, spawning, and death. The multi-target likelihood $g_{k}$ describes the sensor observation process and encapsulates the underlying models of target detection, target-generated measurements, and false alarms.

The multi-target Bayes recursion is used to propagate the posterior distribution $P_{k}\left(\cdot \mid Z_{1: k}\right)$ that describes the current target population based on all the measurements $Z_{1}, \ldots, Z_{k}$ collected so far. The CPHD Bayes recursion aims at simplifying the multi-target Bayes recursion by approximating the predicted and posterior multi-target processes as independent and identically distributed (i.i.d.) processes, ${ }^{5}$ a class of point processes fully characterized by their cardinality distribution $\rho_{\Phi}$ and their intensity measure $\mu_{\Phi}$ [6]. The CPHD filter thus focuses on the propagation of the posterior cardinality distribution $\rho_{k}$ and the posterior intensity measure $\mu_{k}$, rather than the full posterior probability distribution $P_{k}$.

The original construction of the CPHD filter [6] does not consider a target spawning mechanism, and the key contribution of this paper is to propose its integration in the CPHD time prediction equation (see Section III-B). Note that the data update step does not involve the target spawning mechanism and is therefore left out of the scope of this paper. A detailed description of the data update step can be found in [32].

\section{CPHD FILTER PREDICTION With SPAWNING}

This section covers the derivation of the filtering equations for the CPHD filter for various target spawning processes. Section III-A then presents the various models of point processes that will be necessary for the construction of the CPHD filter with spawning in Section III-B.

\section{A. Point Process Models}

1) Bernoulli Process: A Bernoulli process $\Phi$ is characterized by a parameter $0 \leq p \leq 1$ and a spatial distribution $s$. It describes the situation where 1) either there is no object in the scene, or 2) there is a single object in the scene, with state distributed according to $s$. Its projection measures are given by

$$
P_{\Phi}^{(n)}\left(B_{1} \times \ldots \times B_{n}\right)= \begin{cases}1-p, & n=0 \\ p s\left(B_{1}\right), & n=1 \\ 0, & \text { otherwise }\end{cases}
$$

Proposition 5 (p.g.fl. of a Bernoulli process [4]): The p.g.fl. of a Bernoulli process $\Phi$ with parameter $p$ and spatial distribution $s$ is given by

$$
G_{\Phi}(h)=1-p+p \int h(x) s(\mathrm{~d} x) .
$$

In the context of target spawning, a Bernoulli model describes a parent target that may or may not spawn a target at each time step, i.e., between two successive observation scans. It is thus adapted to applications where spawning events are rare with respect to the scan rate of the tracking system, and the operator's knowledge about their relative frequency is described by the parameter $p$. Note, however, that no more than one target may be spawned in a single event.

\footnotetext{
${ }^{5}$ The definition of an i.i.d. process is given in Section III-A.
}

2) Poisson Process: A Poisson process $\Phi$ is characterized by a rate $\lambda \geq 0$ and a spatial distribution $s$. It describes a population whose size follows a Poisson distribution and whose individual states are i.i.d. according to $s$. Its projection measures are given by

$$
P_{\Phi}^{(n)}\left(B_{1} \times \ldots \times B_{n}\right)=e^{-\lambda} \frac{\lambda^{n}}{n !} \prod_{i=1}^{n} s\left(B_{i}\right) .
$$

Proposition 6 (p.g.fl. of a Poisson process [4]): The p.g.fl. of a Poisson process $\Phi$ with rate $\lambda$ and spatial distribution $s$ is given by

$$
G_{\Phi}(h)=\exp \left[\lambda\left(\int h(x) s(\mathrm{~d} x)-1\right)\right] .
$$

In the context of target spawning, a Poisson model describes a parent target spawning a group of targets of various size (including zero or one) at each time step. It is thus adapted to applications where several targets are likely to be spawned from a single spawning event, and the operator's knowledge about the number of spawned targets is described by the rate $\lambda$. Note, however, that the frequency of spawning events cannot be described independently from their size, since the description of an "empty" spawning event (i.e., producing no target) is constrained by the choice of $\lambda$.

3) Zero-Inflated Poisson Process: A zero-inflated Poisson process $\Phi$ (from [33]) is characterized by a parameter $0 \leq p \leq 1$, a rate $\lambda \geq 0$, and a spatial distribution $s$. It describes a population that is 1) either empty, or 2) non-empty, with size following a Poisson distribution and whose individual states are i.i.d. according to $s$. Its projection measures are given by

$$
P_{\Phi}^{(n)}\left(B_{1} \times \ldots \times B_{n}\right)= \begin{cases}1-p+p e^{-\lambda}, & n=0, \\ p e^{-\lambda} \frac{\lambda^{n}}{n !} \prod_{i=1}^{n} s\left(B_{i}\right), & \text { otherwise. }\end{cases}
$$

Note that a Poisson process is a special case of a zero-inflated Poisson process in which the parameter $p$ is set to one.

Proposition 7 (p.g.fl. of a zero-inflated Poisson process): The p.g.fl. of a zero-inflated Poisson process $\Phi$ with parameter $p$, rate $\lambda$, and spatial distribution $s$ is given by

$$
G_{\Phi}(h)=1-p+p \exp \left[\lambda\left(\int h(x) s(\mathrm{~d} x)-1\right)\right] .
$$

In the context of target spawning, a zero-inflated Poisson model "inflates" the occurrence of "empty" spawning events described by a Poisson model. The operator's knowledge about the number of spawned targets is described by the rate $\lambda$, while the frequency of the spawning events is described separately by the parameter $p$. It is thus adapted to many realistic applications where single spawning events are rare with respect to the scan rate of the tracking system, but may produce several targets (e.g., the tracking of a single Earth orbiting satellite in anticipation of a fragmentation event, where one would not expect that it disintegrates into smaller pieces each time it is observed).

4) i.i.d. Process: An i.i.d. process $\Phi$ is characterized by a cardinality distribution $\rho$ and a spatial distribution $s$. It describes a population whose size is distributed according to $\rho$, and whose individual states are i.i.d. according to $s$. Its Janossy measures 
are given by

$$
J_{\Phi}^{(n)}\left(B_{1} \times \ldots \times B_{n}\right)=n ! \rho(n) \prod_{i=1}^{n} s\left(B_{i}\right) .
$$

Note that a Poisson process is a special case of i.i.d. process in which the cardinality distribution $\rho$ is Poisson.

\section{B. Prediction Step}

In this section, we propose an alternative expression of the original CPHD time prediction step [6] in which newborn targets may originate from either a spawning mechanism or a spontaneous birth. Note that the assumptions on the posterior multi-target process from the previous time step, the target survival mechanism, the spontaneous birth mechanism, and the target evolution mechanism are identical to the original assumptions in [6].

Theorem 1 (CPHD with spawning: prediction step): Assuming that, at step $k$ :

- The posterior multi-target process $\Phi_{k-1}$ is an i.i.d. process with intensity measure $\mu_{k-1}$, with cardinality distribution $\rho_{k-1}$, and spatial distribution $s_{k-1}$,

- A target in state $x$ at time $k-1$ survived to time $k$ with probability $p_{\mathrm{s}, k}(x)$,

- A surviving target in state $x$ at time $k-1$ evolved since time $k-1$ according to a Markov transition $f_{\mathrm{s}, k}(\cdot \mid x)$,

- Newborn targets are born, independently from prior targets, following a process with intensity measure $\mu_{\gamma, k}$, and cardinality distribution $\rho_{\gamma, k}$,

- Newborn targets were spawned from a prior target, in state $x$ at time $k-1$, following a process with intensity measure $\mu_{\mathrm{b}, k}(\cdot \mid x)$, and cardinality distribution $\rho_{\mathrm{b}, k}(\cdot \mid x)$,

then the intensity measure $\mu_{k \mid k-1}$ and cardinality distribution $\rho_{k \mid k-1}$ of the predicted multi-target process $\Phi_{k \mid k-1}$ are given by

$$
\begin{aligned}
\mu_{k \mid k-1}(\cdot)= & \int\left[p_{\mathrm{s}, k}(x) f_{\mathrm{s}, k}(\cdot \mid x)\right. \\
& \left.+\mu_{\mathrm{b}, k}(\cdot \mid x)\right] \mu_{k-1}(\mathrm{~d} x)+\mu_{\gamma, k}(\cdot), \\
\rho_{k \mid k-1}(n)= & \sum_{q=0}^{n} \rho_{\gamma, k}(n-q) \sum_{j=0}^{q} B_{q, j}\left(b_{1}, \ldots, b_{q}\right) \\
& \times\left[\sum_{m \geq j} \frac{m !}{q !(m-j) !} \rho_{k-1}(m) b_{0}{ }^{m-j}\right],
\end{aligned}
$$

where $B_{q, j}$ is the partial Bell polynomial [13, p. 412] given by equation (32) as shown at the bottom of this page and where the coefficients $b_{i}$ are given by

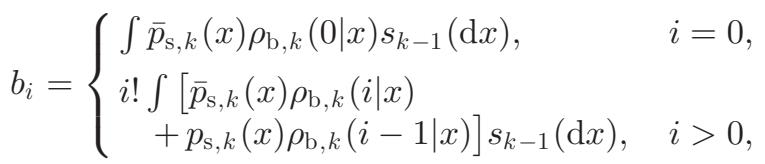

where $\bar{p}_{\mathrm{s}, k}(\cdot) \equiv 1-p_{\mathrm{s}, k}(\cdot)$.

The proof is given in the Appendix. Note that the structure of the predicted cardinality (31) allows for its efficient computation through an algorithm dedicated to the computation of partial Bell polynomials. Exploiting the recursive formula [13, (11.11)], we propose in Section VI an implementation of the predicted cardinality (31) with a computational cost of $\mathcal{O}\left(n_{\max }^{3}\right)$, where $n_{\max }$ is the maximum number of targets considered for the support of the cardinality distributions. Also, note that the construction of the predicted intensity (30) is identical to that of the original PHD filter (see [34], for example).

Corollary 1: The intensity measure $\mu_{\mathrm{b}, k}$ and the coefficients $b_{i}$, describing the spawning process in the CPHD prediction step (30), (31), depend on the modeling choices. Denoting $\bar{p}_{\mathrm{b}, k}(\cdot) \equiv 1-p_{\mathrm{b}, k}(\cdot)$, they are given as follows for various spawning processes:

a) Bernoulli process, with parameter $p_{\mathrm{b}, k}$ and spatial distribution $s_{\mathrm{b}, k}$ :

$$
\mu_{\mathrm{b}, k}(\cdot \mid x)=p_{\mathrm{b}, k}(x) s_{\mathrm{b}, k}(\cdot \mid x)
$$

and

$$
b_{i}= \begin{cases}\int \bar{p}_{\mathrm{s}, k}(x) \bar{p}_{\mathrm{b}, k}(x) s_{k-1}(\mathrm{~d} x), & i=0 \\ \int\left[p_{\mathrm{s}, k}(x) \bar{p}_{\mathrm{b}, k}(x)+\bar{p}_{\mathrm{s}, k}(x) p_{\mathrm{b}, k}(x)\right] s_{k-1}(\mathrm{~d} x), & i=1 \\ 2 \int p_{\mathrm{s}, k}(x) p_{\mathrm{b}, k}(x) s_{k-1}(\mathrm{~d} x), & i=2 \\ 0, & i>2\end{cases}
$$

b) Poisson process, with rate $\lambda_{\mathrm{b}, k}$ and spatial distribution $s_{\mathrm{b}, k}$ :

$$
\mu_{\mathrm{b}, k}(\cdot \mid x)=\lambda_{\mathrm{b}, k}(x) s_{\mathrm{b}, k}(\cdot \mid x)
$$

and

$$
b_{i}=\int \lambda_{\mathrm{b}, k}^{i-1}(x) e^{-\lambda_{\mathrm{b}, k}(x)}\left[\bar{p}_{\mathrm{s}, k}(x) \lambda_{\mathrm{b}, k}(x)+i p_{\mathrm{s}, k}(x)\right] s_{k-1}(\mathrm{~d} x),
$$

for $i \geq 0$.

c) zero-inflated Poisson process, with parameter $p_{\mathrm{b}, k}$, rate $\lambda_{\mathrm{b}, k}$, and spatial distribution $s_{\mathrm{b}, k}$ :

$$
\mu_{\mathrm{b}, k}(\cdot \mid x)=p_{\mathrm{b}, k}(x) \lambda_{\mathrm{b}, k}(x) s_{\mathrm{b}, k}(\cdot \mid x),
$$

and, equation (39) as shown at the bottom of this page.

$$
B_{q, k}\left(x_{1}, x_{2}, \ldots, x_{q}\right)=\sum_{\substack{k_{1}+2 k_{2}+\cdots+q k_{q}=q \\ k_{1}+k_{2}+\cdots+k_{q}=k}} \frac{q !}{k_{1} !(1 !)^{k_{1}} k_{2} !(2 !)^{k_{2}} \cdots k_{q} !(q !)^{k_{q}}} x_{1}^{k_{1}} x_{2}^{k_{2}} \cdots x_{q}^{k_{q}}
$$

$$
b_{i}= \begin{cases}\int \bar{p}_{\mathrm{s}, k}(x)\left[\bar{p}_{\mathrm{b}, k}(x)+p_{\mathrm{b}, k}(x) e^{-\lambda_{\mathrm{b}, k}(x)}\right] s_{k-1}(\mathrm{~d} x) & i=0 \\ \int\left[\bar{p}_{\mathrm{s}, k}(x) p_{\mathrm{b}, k}(x) e^{-\lambda_{\mathrm{b}, k}(x)} \lambda_{\mathrm{b}, k}(x)+p_{\mathrm{s}, k}(x)\left[\bar{p}_{\mathrm{b}, k}(x)+p_{\mathrm{b}, k}(x) e^{-\lambda_{\mathrm{b}, k}(x)}\right]\right] s_{k-1}(\mathrm{~d} x), & i=1 \\ \int p_{\mathrm{b}, k}(x) \lambda_{\mathrm{b}, k}^{i-1}(x) e^{-\lambda_{\mathrm{b}, k}(x)}\left[\bar{p}_{\mathrm{s}, k}(x) \lambda_{\mathrm{b}, k}(x)+i p_{\mathrm{s}, k}(x)\right] s_{k-1}(\mathrm{~d} x), & i \geq 2\end{cases}
$$


The proof is given in the Appendix. Note that the construction of the predicted cardinality for a CPHD filter with Bernoulli and Poisson spawning processes was previously explored in [14], following traditional Bayesian statistics. The results provided in [14] are not supported by a detailed construction; however, an earlier work by the same authors [15] proposed a more principled derivation of the predicted cardinality through the exploitation of p.g.fs. It can be shown (a sketch is given in the Appendix) that the general expression of the predicted cardinality $[15$, (A.15)] is equivalent to our presentation through the Bell polynomials in Eq. (31). However, we believe that the latter facilitates practical implementation of the CPHD filter with spawning, and allows for a clear presentation of the exact time prediction equation (i.e., without requiring additional approximations) for specific models of spawning through the coefficients $b_{i}$, as illustrated in Corollary 1 .

\section{Simulation}

In this section we illustrate the CPHD filter with spawning models through a simulation-based scenario. The Gaussian Mixture (GM) implementation of the CPHD filter is briefly described in Section IV-A, followed by a description of the metrics exploited for the analysis of the filter results in Section IV-B. The scenario and the selection of the filter parameters are detailed in Section IV-C, and the results are discussed in Section IV-D.

\section{A. The GM-CPHD Filter With Spawning}

Since the incorporation of spawning in the CPHD filtering process does not affect the data update step, we shall focus in this section on the specifics of the prediction step for the GM-CPHD filter with spawning. A description of the usual GM-CPHD, including the implementation of the spontaneous birth term, is given in [32].

1) Filtering Assumptions: We follow the usual assumptions of the GM-CPHD filter [32] regarding the transition process from time $k-1$ to time $k$, namely, that the probability of survival $p_{\mathrm{s}, k}$ is uniform over the state space $\mathbf{X}$ and the transition $f_{\mathrm{s}, k}$ follows a linear Gaussian dynamical model:

$$
\begin{aligned}
p_{\mathrm{s}, k}(\cdot) & \equiv p_{\mathrm{s}, k}, \\
f_{\mathrm{s}, k \mid k-1}(\cdot \mid x) & =\mathcal{N}\left(\cdot ; F_{k} x, Q_{k}\right),
\end{aligned}
$$

where $\mathcal{N}(\cdot ; m, P)$ denotes a Gaussian distribution with mean $m$ and covariance $P, F_{k}$ is a state transition matrix, and $Q_{k}$ is a process noise covariance matrix.

Regardless of the chosen spawning model (see Theorem 1), we further assume that the spatial distribution of each spawned object $s_{\mathrm{b}, k}$ can be described as the Gaussian mixture

$$
s_{\mathrm{b}, k}(\cdot \mid x)=\sum_{j=1}^{J_{\mathrm{b}, k}} w_{\mathrm{b}, k}^{(j)} \mathcal{N}\left(\cdot ; F_{\mathrm{b}, k}^{(j)} x+d_{\mathrm{b}, k}^{(j)}, Q_{\mathrm{b}, k}^{(j)}\right),
$$

where $d_{\mathrm{b}, k}^{(j)}$ is a deviation vector, $F_{\mathrm{b}, k}^{(j)}$ is a spawning transition matrix, and $Q_{\mathrm{b}, k}^{(j)}$ is a spawning noise covariance matrix, for $1 \leq j \leq J_{\mathrm{b}, k}$, and $\sum_{j=1}^{J_{\mathrm{b}, k}} w_{\mathrm{b}, k}^{(j)}=1$. Also, we assume that the model parameters $p_{\mathrm{b}, k}, \lambda_{\mathrm{b}, k}$, when applicable, are uniform over the state space $\mathbf{X}$ :

$$
\begin{aligned}
p_{\mathrm{b}, k}(\cdot) & \equiv p_{\mathrm{b}, k}, \\
\lambda_{\mathrm{b}, k}(\cdot) & \equiv \lambda_{\mathrm{b}, k} .
\end{aligned}
$$

2) Predicted Intensity: The construction of the predicted intensity $\mu_{k \mid k-1}$ in Eq. (30) follows a similar structure as for the usual GM-CPHD filter [34]. Assume that the posterior intensity $\mu_{k-1}$ can be written as a Gaussian mixture of the form

$$
\mu_{k-1}(\cdot)=\sum_{j=1}^{J_{k-1}} w_{k-1}^{(j)} \mathcal{N}\left(\cdot ; m_{k-1}^{(j)}, P_{k-1}^{(j)}\right),
$$

where $m_{k-1}^{(j)}$ (resp. $P_{k-1}^{(j)}$ ) is the posterior mean (resp. covariance) of the $j$ th component of the mixture. Then the predicted intensity $\mu_{k \mid k-1}$ can also be written as a Gaussian mixture of the form

$$
\mu_{k \mid k-1}(\cdot)=\mu_{\mathrm{s}, k \mid k-1}(\cdot)+\mu_{\mathrm{b}, k \mid k-1}(\cdot),
$$

where the surviving component $\mu_{\mathrm{s}, k \mid k-1}$ is the Gaussian mixture

$$
\mu_{\mathrm{s}, k \mid k-1}(\cdot)=p_{\mathrm{s}, k} \sum_{j=1}^{J_{k-1}} w_{k-1}^{(j)} \mathcal{N}\left(\cdot ; m_{\mathrm{s}, k \mid k-1}^{(j)}, P_{\mathrm{s}, k \mid k-1}^{(j)}\right),
$$

with

$$
\begin{aligned}
m_{\mathrm{s}, k \mid k-1}^{(j)} & =F_{k} m_{k-1}^{(j)}, \\
P_{\mathrm{s}, k \mid k-1}^{(j)} & =Q_{k}+F_{k} P_{k-1}^{(j)} F_{k}^{T},
\end{aligned}
$$

for $1 \leq j \leq J_{k-1}$, and the spawning component $\mu_{\mathrm{b}, k \mid k-1}$ is the Gaussian mixture

$$
\begin{aligned}
& \mu_{\mathrm{b}, k \mid k-1}(\cdot)=\alpha_{\mathrm{b}, k} \sum_{j=1}^{J_{k-1}} w_{k-1}^{(j)} \\
& \times \sum_{i=1}^{J_{\mathrm{b}, k}} w_{\mathrm{b}, k}^{(i)} \mathcal{N}\left(\cdot ; m_{b, k \mid k-1}^{(j, i)}, P_{b, k \mid k-1}^{(j, i)}\right),
\end{aligned}
$$

with

$$
\begin{aligned}
m_{\mathrm{b}, k \mid k-1}^{(j, i)} & =F_{\mathrm{b}, k}^{(i)} m_{k-1}^{(j)}+d_{\mathrm{b}, k}^{(i)}, \\
P_{\mathrm{b}, k \mid k-1}^{(j, i)} & =Q_{\mathrm{b}, k}^{(i)}+F_{\mathrm{b}, k}^{(i)} P_{k-1}^{(j)}\left(F_{\mathrm{b}, k}^{(i)}\right)^{T},
\end{aligned}
$$

for $1 \leq j \leq J_{k-1}, 1 \leq i \leq J_{\mathrm{b}, k}$, and the scalar $\alpha_{\mathrm{b}, k}$ depends on the spawning model:

$$
\alpha_{\mathrm{b}, k}= \begin{cases}p_{\mathrm{b}, k}, & \text { Bernoulli process, } \\ \lambda_{\mathrm{b}, k}, & \text { Poisson process, } \\ p_{\mathrm{b}, k} \lambda_{\mathrm{b}, k}, & \text { zero-inflated Poisson process. }\end{cases}
$$

3) Predicted Cardinality Distribution: Due to the assumptions presented in Section IV-A1, the coefficients of the Bell polynomial in Eq. (31) have the simpler form

a) Bernoulli process:

$$
b_{i}= \begin{cases}\left(1-p_{\mathrm{s}, k}\right)\left(1-p_{\mathrm{b}, k}\right), & i=0, \\ p_{\mathrm{s}, k}\left(1-p_{\mathrm{b}, k}\right)+\left(1-p_{\mathrm{s}, k}\right) p_{\mathrm{b}, k}, & i=1 \\ 2 p_{\mathrm{s}, k} p_{\mathrm{b}, k}, & i=2 \\ 0, & i>2\end{cases}
$$


b) Poisson process:

$$
b_{i}=\lambda_{\mathrm{b}, k}^{i-1} e^{-\lambda_{\mathrm{b}, k}}\left[\left(1-p_{\mathrm{s}, k}\right) \lambda_{\mathrm{b}, k}+i p_{\mathrm{s}, k}\right], \quad i \geq 0 .
$$

c) zero-inflated Poisson process:

$$
b_{i}= \begin{cases}\left(1-p_{\mathrm{s}, k}\right)\left(1-p_{\mathrm{b}, k}+p_{\mathrm{b}, k} e^{-\lambda_{\mathrm{b}, k}}\right), & i=0 \\ \left(1-p_{\mathrm{s}, k}\right) p_{\mathrm{b}, k} e^{-\lambda_{\mathrm{b}, k}} \lambda_{\mathrm{b}, k} & \\ \quad+p_{\mathrm{s}, k}\left(1-p_{\mathrm{b}, k}+p_{\mathrm{b}, k} e^{-\lambda_{\mathrm{b}, k}}\right), & i=1, \\ p_{\mathrm{b}, k} \lambda_{\mathrm{b}, k}^{i-1} e^{-\lambda_{\mathrm{b}, k}}\left[\left(1-p_{\mathrm{s}, k}\right) \lambda_{\mathrm{b}, k}+i p_{\mathrm{s}, k}\right], & i \geq 2 .\end{cases}
$$

The predicted cardinality distribution is then computed by the appropriate substitution of Eqs. (53)-(55) into Eq. (31).

\section{B. Evaluation Metrics}

To compare the multi-target state representing the true targets in the scene - the "ground truth" - and a collection of targets extracted from the filter's output, we exploit the optimal subpattern assignment (OSPA) metric [35] for assessing the accuracy of multi-object filters. Given two sets $X=\left\{x_{1}, \ldots, x_{m}\right\}$, $x_{i} \in \mathbf{X}, \quad 1 \leq i \leq m, \quad$ and $\quad Y=\left\{y_{1}, \ldots, y_{n}\right\}, \quad y_{j} \in \mathbf{X}$, $1 \leq j \leq n$, the second-order OSPA distance $d_{2}^{(c)}(X, Y)$ between $X$ and $Y$ is defined as

$$
\begin{aligned}
& d_{2}^{(c)}(X, Y)= \\
& \begin{cases}0, & m=n=0, \\
{\left[\frac{1}{n}\left(\min _{\pi \in \Pi_{n}} \sum_{i=1}^{m} d^{(c)}\left(x_{i}, y_{\pi(i)}\right)^{2}+c^{2}(n-m)\right)\right]^{1 / 2}} & m \leq n,(56) \\
d_{2}^{(c)}(Y, X), & \text { otherwise, }\end{cases}
\end{aligned}
$$

with

$$
d^{(c)}\left(x_{i}, y_{j}\right)=\min \left(c,\left\|x_{i}-y_{j}\right\|\right)
$$

where $c$ is the cutoff parameter, and $\|\cdot\|$ is the usual norm on $\mathbf{X}$. The OSPA distance is such that $0 \leq d_{2}^{(c)}(X, Y) \leq c$; $d_{2}^{(c)}(X, Y)=0$ indicates that $X$ and $Y$ are identical, while $d_{2}^{(c)}(X, Y)$ increases with the discrepancies between $X$ and $Y$, taking into account mismatches in number of elements and element states.

In order to compare the true number of targets in the scene and a estimated cardinality distribution extracted from the filter's output, we exploit the Hellinger distance [36]. Given two finite cardinality distributions $P=\left(p_{1}, \ldots, p_{k}\right)$ and $Q=$ $\left(q_{1}, \ldots, q_{k}\right)$, the Hellinger distance $d_{H}(P, Q)$ is

$$
d_{H}(P, Q)=\frac{1}{\sqrt{2}} \sqrt{\sum_{i=1}^{k}\left(\sqrt{p_{i}}-\sqrt{q_{i}}\right)^{2}} .
$$

Note that in (58), the coefficient $1 / \sqrt{2}$ is included in order to scale the Hellinger distance such that it is bounded as $0 \leq d_{H}(P, Q) \leq 1 ; d_{H}(P, Q)=0$ indicates that $P$ and $Q$ are equivalent, where as $d_{H}(P, Q) \rightarrow 1, P$ and $Q$ become increasingly dissimilar.

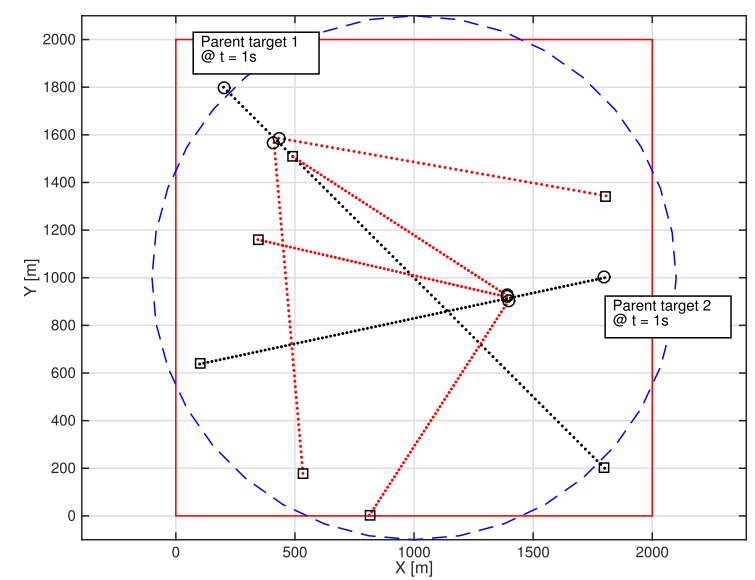

Fig. 1. Target trajectories. A circle " $\bigcirc$ " indicates where a trajectory begins, and a square " $\square$ " indicates where a trajectory ends. The large square indicates the limits of the sensor's field of view (FoV) and the large dashed circle represents the $90 \%$ confidence region of the Gaussian component of the spontaneous birth model.

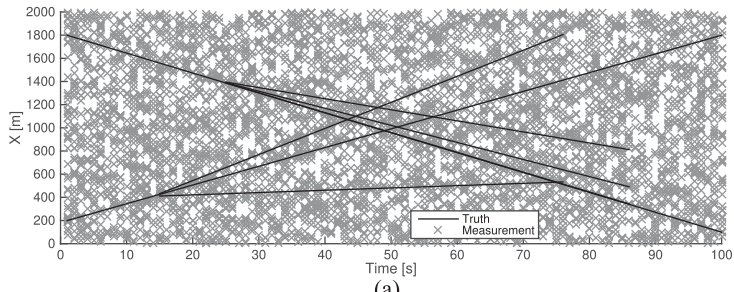

(a)

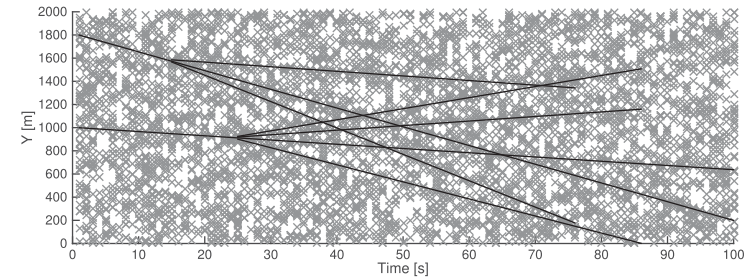

(b)

Fig. 2. Collected measurements (gray crosses) and target positions (black lines). (a) $\mathrm{x}$-axis. (b) $\mathrm{y}$-axis.

\section{Scenario and Filter Setup}

A point $[x, y, \dot{x}, \dot{y}]$ of the single-target state space $\mathbf{X} \subset \mathbb{R}^{4}$ describes the position and velocity coordinates of an object in a square surveillance region of size $2000 \mathrm{~m} \times 2000 \mathrm{~m}$. The simulated multi-target tracking scenario consists of one scan per second for $100 \mathrm{~s}$, and up to seven targets evolving in the region with constant velocity. Two targets are present at the beginning of the scenario and each spawns targets at different times: target 1 spawns two additional targets at $t=15 \mathrm{~s}$ and target 2 spawns three additional targets at $t=25 \mathrm{~s}$. All spawned targets have a lifespan of $60 \mathrm{~s}$. Fig. 1 shows the trajectories of the targets cumulated over time, while Fig. 2 illustrates these trajectories and the collected measurements across time.

The probability of survival $p_{\mathrm{s}, k}$ (40) is constant throughout the scenario, and set to $p_{\mathrm{s}, k}=0.99$. The target motion model $f_{\mathrm{s}, k \mid k-1}(41)$ is set as follows:

$$
F_{k}=\left[\begin{array}{cc}
\mathbf{1}_{2} & \Delta \mathbf{1}_{2} \\
\mathbf{0}_{2} & \mathbf{1}_{2}
\end{array}\right], \quad Q_{k}=\sigma_{\nu}^{2}\left[\begin{array}{cc}
\frac{\Delta^{4}}{4} \mathbf{1}_{2} & \frac{\Delta^{3}}{2} \mathbf{1}_{2} \\
\frac{\Delta^{3}}{2} \mathbf{1}_{2} & \Delta^{2} \mathbf{1}_{2}
\end{array}\right],
$$


TABLE I

SPAWN MOdEL PARAMETERS

\begin{tabular}{cccc}
\hline Model & $p_{\mathrm{b}, k}$ & $\lambda_{\mathrm{b}, k}$ & $\mu_{\mathrm{b}, k}(\cdot \mid x)$ \\
\hline Bernoulli & 0.01 & - & $0.01 \mathcal{N}\left(\cdot ; x, Q_{\mathrm{b}, k}\right)$ \\
Poisson & - & 0.025 & $0.025 \mathcal{N}\left(\cdot ; x, Q_{\mathrm{b}, k}\right)$ \\
zero-inflated Poisson & 0.01 & 2.5 & $0.025 \mathcal{N}\left(\cdot ; x, Q_{\mathrm{b}, k}\right)$
\end{tabular}

where $\Delta=1 \mathrm{~s}, \sigma_{\nu}=5 \mathrm{~ms}^{-2}$, and $\mathbf{1}_{n}$ (resp. $\mathbf{0}_{n}$ ) denotes the $n \times n$ identity (resp. zero) matrix.

The sensor's probability of detection is uniform over the sensor's FoV, and set at a constant value of 0.95 throughout the scenario. Each target-generated measurement consists of the target's coordinate position with an independent Gaussian white noise on each component, with a standard deviation of $10 \mathrm{~m}$. Spurious measurements are modeled as a Poisson point process with uniform spatial distribution over the state space and an average number of clutter per unit volume of $12.5 \times 10^{-6} \mathrm{~m}^{-2}$, that is, an average of 50 clutter returns per scan over the surveillance region.

For the sake of comparison, the usual GM-CPHD filter [32] with spontaneous birth and no spawning is implemented as well. The spontaneous birth model is Poisson, with a constant rate of 0.025 per time step (which yields, over the $100 \mathrm{~s}$ of the scenario, an average of 2.5 newborn targets). The spatial distribution is modeled with a single Gaussian component, centered on the sensor's FoV as illustrated in Fig. 1.

The spatial distribution of the spawning (42) is identical for the three considered models. We assume no spawned target deviation vectors, and a standard deviation of 12 units is set on each component of the spawning noise covariance, i.e.,

$$
F_{\mathrm{b}, k}=\left[\begin{array}{cc}
\mathbf{1}_{2} & \mathbf{0}_{2} \\
\mathbf{0}_{2} & \mathbf{1}_{2}
\end{array}\right], d_{\mathrm{b}, k}=\mathbf{0}, Q_{\mathrm{b}, k}=\left[\begin{array}{cc}
\sigma_{\mathrm{b}}^{2} \mathbf{1}_{2} & \mathbf{0}_{2} \\
\mathbf{0}_{2} & \dot{\sigma}_{\mathrm{b}}^{2} \mathbf{1}_{2}
\end{array}\right],
$$

where $\mathbf{0}$ denotes the null vector in $\mathbf{X}, \sigma_{\mathrm{b}}=12 \mathrm{~m}$, and $\dot{\sigma}_{\mathrm{b}}=$ $12 \mathrm{~ms}^{-1}$.

The parameters of the three spawning models are set as follows. The zero-inflated Poisson model assumes one spawning per parent target during the scenario with an average of 2.5 daughter targets per spawning event, thus $p_{\mathrm{b}, k}$ and $\lambda_{\mathrm{b}}$ are set to 0.01 and 2.5 , respectively. Relative to the zero-inflated Poisson model, the Poisson model is set to yield a similar spawning intensity thus its $\lambda_{\mathrm{b}, k}$ is set to 0.025 , whereas the Bernoulli model is set to yield a similar spawning frequency so its $p_{\mathrm{b}, k}$ is set to 0.01 . These parameters are also presented in Table I.

It is interesting to note that neither the Poisson nor the Bernoulli models are equipped to capture the nature of the spawning events occurring in this scenario, since, per construction, the Poisson model is a poor match for spawning events occuring at unknown dates and the Bernoulli model is a poor match for spawning events creating more than one daughter target. The zero-inflated Poisson model possesses a greater flexibility and should be able to cope with a wider range of spawning situations; in any case, it is expected to yield better performances on the scenario presented in this paper.

To maintain tractability, GM components are truncated with threshold $T=10^{-5}$, pruned with maximum number of components $J_{\max }=100$, and merged with threshold $U=4$ (see [34] for more details on the pruning and merging mechanisms). Ad-

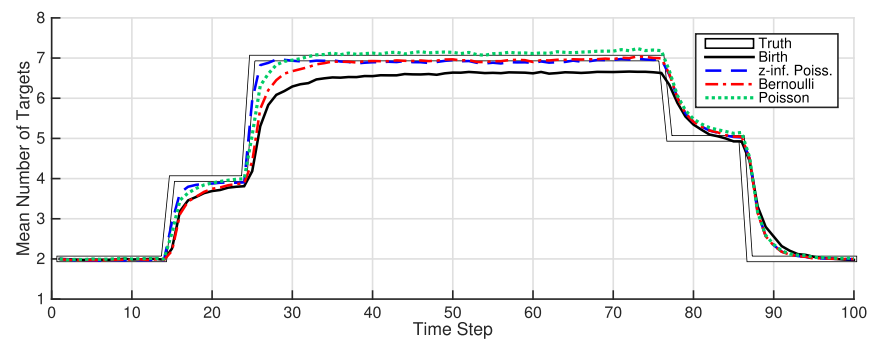

Fig. 3. MAP estimate of the number of targets (averaged on 500 runs).

ditionally, the maximum number of targets is set to $N_{\max }=20$ to circumvent issues with infinitely tailed cardinality distributions [32].

\section{Simulation Results}

The proposed spawning models and the birth model are implemented with the GM-CPHD filter, and compared over 500 Monte Carlo (MC) runs of the multi-target scenario described in Section IV-C.

The MAP estimate of the number of targets is plotted in Fig. 3, along with the true number of targets in the scene. The results suggest that the spawning models provide a better estimate of the number of targets and, in particular, converge faster to the true number of targets following the appearance of new targets in the scene. This is expected, because the scenario does not feature any spontaneous but only spawning-related births, and thus in this context spawning models are a better match than the birth model.

Among the three spawning models, the zero-inflated Poisson converges the fastest following the appearance of new targets, while the Bernoulli model converges the slowest. This is expected, for the zero-inflated Poisson model provides the best match to the spawning events occurring in this scenario. Note in particular that the Bernoulli model may not consider the appearance of more than one daughter per spawning event, and must therefore stage the multiple-target appearances across several successive time steps; in other words, the Bernoulli is illadapted to "busy" events where targets appear simultaneously. Note also the slight overestimation shown by the Poisson model when the true number of target is stable. Per construction, the Poisson model is well-equipped for the simultaneous appearance of an arbitrary number of spawned targets at any time step, but it fails at coping with "quiet" periods where no spawning occurs because, unlike the zero-inflated Poisson model, it does not temper the Poisson-driven spawning with a probability of spawning. In other words, the Poisson model is ill-adapted to the spawning events shown in this scenario.

Note that all models - spawning and birth - follow the same mechanism for target deaths and yield much closer performances when target disappearances occur.

Similar conclusions can be drawn from the comparisons of the OSPA distances shown in Fig. 4. All models show error spikes at times of spawning $(t=15 \mathrm{~s}, t=25 \mathrm{~s})$ and death $(t=76 \mathrm{~s}$, $t=86 \mathrm{~s}$ ), however, the spawning models recover more quickly than the birth model, and have consistently lower errors.

The quality of the estimation of the number of targets proposed by the four models is further illustrated in Fig. 5, where the Hellinger distance between the cardinality distribution prop- 


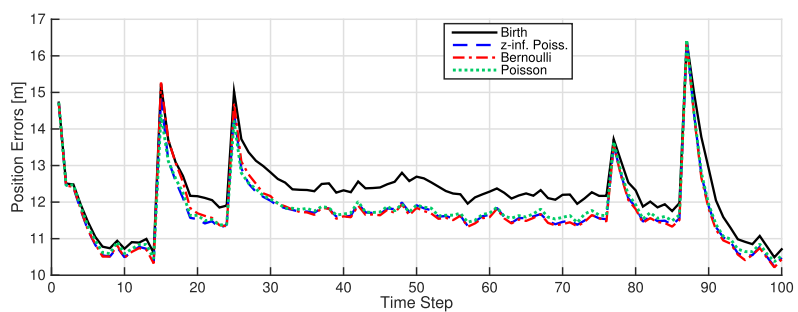

(a)

(b)

Fig. 4. OSPA distance (averaged on 500 runs). (a) Position. (b) Velocity.

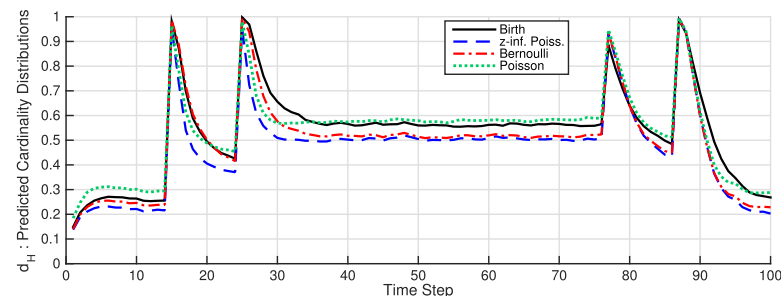

(a)

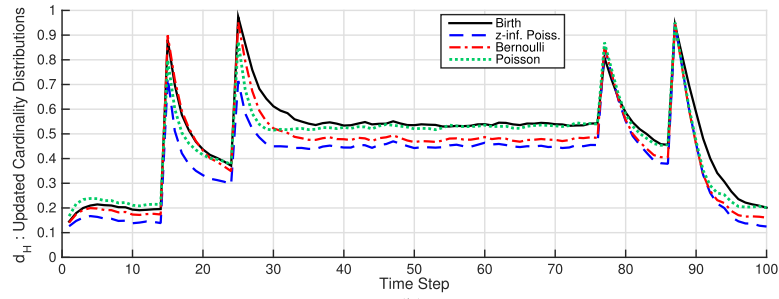

(b)

Fig. 5. Hellinger distances (averaged on 500 runs). (a) Predicted cardinality. (b) Updated cardinality.

agated by each model and the "ideal" cardinality distribution (i.e., a distribution in which all the mass is concentrated on the true number of targets).

The results in Fig. 5 allow a more refined analysis of the proposed models. All the models yield poor estimates immediately after a change in the true number of targets, ${ }^{6}$ but the zero-inflated Poisson model converges the fastest following a target birth/death and it converges to the best estimate during periods where the number of target is stable. The Poisson model converges faster than the Bernoulli model, but to a worse estimate: this is expected, since the Poisson model is ill-adapted to "quiet" periods while the Bernoulli model is ill-adapted to "busy" events (see discussion above on Fig. 3).

As expected, the updated cardinality distributions are consistently more accurate than the predicted cardinality distributions since they benefit from the processing of an additional measurement batch.

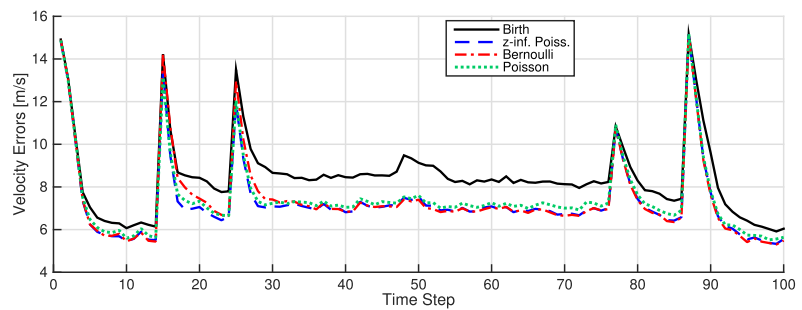

\section{CONCLUSION}

The motivation for the work presented in this paper is the resolution of multi-object detection and tracking problems in which newborn objects are spawned from preexisting ones. To this end, the construction of a CPHD filter in which the appearance of newborn targets is modeled with a spawning mechanism rather than spontaneous birth is proposed, based on a principled derivation procedure within the FISST framework.

A GM implementation of the CPHD filter with spawning is then presented, considering three different models for the spawning mechanism based on a Bernoulli, a Poisson, or a zero-inflated Poisson process. The three resulting filters are then illustrated, analyzed, and compared to a usual CPHD filter with spontaneous birth but no spawning, on the same simulated scenario involving two parent targets spawning a total of five daughter targets. Results show that a spawning model, appropriately chosen for a given application, can provide better estimates than a spontaneous birth model.

\section{ALGORITHMS}

\section{Algorithm 1: Cardinality prediction (time $k$ ).}

\section{Input}

Posterior cardinality distribution: $\rho_{k-1}$

Posterior spatial distribution: $s_{k-1}$

\section{Parameters}

Cardinality distribution of spawning: $\rho_{\mathrm{b}, k}$

Cardinality distribution of spontaneous birth: $\rho_{\gamma, k}$

Probability of survival: $p_{\mathrm{s}, k}$

\section{Initialisation}

for $n \in\left[0 n_{\text {max }}\right]$ do

Predicted card. dist.: $\rho_{k \mid k-1}(n):=0$

Mult. coefficient: $c_{n}:=\sum_{m=n}^{n_{\max }} \frac{m !}{(m-n) !} \rho_{k-1}(m) b_{0}^{m-n}$

Bell polynomial: $B_{n, 0}:=[n==0]$

Bell coefficient: $b_{n}:=$ (see Eq. (33))

Bell sum: $B_{n}:=B_{n, 0} c_{0}$

\section{end for}

\section{Prediction}

for $n \in\left[0 n_{\max }\right]$ do

for $m \in[1 n]$ do

Bell pol.: $B_{n, m}:=\sum_{q=m-1}^{n-1}\left(\begin{array}{c}n-1 \\ n-1-q\end{array}\right) b_{n-q} B_{q, m-1}$

Bell sum: $B_{n}+=B_{n, m} c_{m}$

\section{end for}

Bell sum: $B_{n} \times=\frac{1}{n !}$

for $m \in\left[0 n_{\max }-n\right]$ do

Predicted card. dist.: $\rho_{k \mid k-1}(n+m)+=\rho_{\gamma, k}(m) B_{n}$ end for

\section{end for}

\section{Output}

${ }^{6}$ Recall from Eq. (58) that the Hellinger distance $d_{H}$ is such that $0 \leq d_{H} \leq 1$.

Predicted cardinality distribution: $\rho_{k \mid k-1}$ 


\section{APPENDIX}

\section{A. Proof of Theorem 1}

For the sake of simplicity, the time subscripts will be omitted throughout the proof when there is no ambiguity.

1) Predicted p.g.fl.: Let us focus first on the p.g.fl. $G_{k \mid k-1}$ of the predicted multi-target point process $\Phi_{k \mid k-1}$. Each parent target in the population, represented by the prior point process $\Phi_{k-1}$, generates daughter targets in the predicted population in two ways:

- a daughter target stemming from the (eventual) survival of the parent target, represented by a survival point process $\Phi_{\mathrm{S}}$,

- a population of daughter spawned from the parent target, represented by a spawning point process $\Phi_{\mathrm{b}}$.

Using Eq. (8), and denoting by $G_{\mathrm{S}}$ (resp. $G_{\mathrm{b}}$ ) the p.g.fl. of the survival (resp. spawning) point process, we can describe the evolution of a parent target with state $x \in \mathbf{X}$ with a compound process with p.g.fl.

$$
G_{\mathrm{c}}(h \mid x)=G_{\mathrm{s}}(h \mid x) G_{\mathrm{b}}(h \mid x) .
$$

In addition, a population of newborn targets is generated independently of the prior targets, represented by a spontaneous birth process $\Phi_{\gamma}$ whose p.g.fl. is denoted by $G_{\gamma}$. Exploiting the Galton-Watson equation (9), we may finally write

$$
\begin{aligned}
G_{k \mid k-1}(h) & =G_{k-1}\left(G_{\mathrm{c}}(h \mid \cdot)\right) G_{\gamma}(h) \\
& =G_{k-1}\left(G_{\mathrm{s}}(h \mid \cdot) G_{\mathrm{b}}(h \mid \cdot)\right) G_{\gamma}(h)
\end{aligned}
$$

2) Predicted Intensity: Let us now focus on the expression of the predicted intensity $\mu_{k \mid k-1}$. For that, let us fix an arbitrary measurable subset $B \in \mathbf{B}_{\mathbf{X}}$. The expression of the intensity evaluated in $B$ can be recovered from the first derivative of the p.g.fl. $G_{k \mid k-1}$ using Eq. (20):

$$
\begin{aligned}
\mu_{k \mid k-1}(B) & =\left.\delta G_{k \mid k-1}\left(h ; 1_{B}\right)\right|_{h=1} \\
& =\left.\delta\left(G_{k-1}\left(G_{\mathrm{c}}(h \mid \cdot)\right) G_{\gamma}(h) ; 1_{B}\right)\right|_{h=1}
\end{aligned}
$$

Using the product rule (14) it becomes

$$
\begin{aligned}
\mu_{k \mid k-1}(B)= & \left.\delta\left(G_{k-1}\left(G_{\mathrm{c}}(h \mid \cdot)\right) ; 1_{B}\right)\right|_{h=1} \underbrace{G_{\gamma}(1)}_{=1} \\
& +\left.\underbrace{G_{k-1}\left(G_{\mathrm{c}}(1 \mid \cdot)\right)}_{=1} \delta G_{\gamma}\left(h ; 1_{B}\right)\right|_{h=1}
\end{aligned}
$$

Using the definition of the p.g.fl. (5a) then yields

$$
\begin{aligned}
\mu_{k \mid k-1}(B)= & \left.\delta\left(\int_{\mathcal{X}}\left[\prod_{x \in \varphi} G_{\mathrm{c}}(h \mid x)\right] P_{k-1}(\mathrm{~d} \varphi) ; 1_{B}\right)\right|_{h=1} \\
& +\left.\delta G_{\gamma}\left(h ; 1_{B}\right)\right|_{h=1} \\
= & \left.\int_{\mathcal{X}} \delta\left(\prod_{x \in \varphi} G_{\mathrm{c}}(h \mid x) ; 1_{B}\right)\right|_{h=1} P_{k-1}(\mathrm{~d} \varphi) \\
& +\left.\delta G_{\gamma}\left(h ; 1_{B}\right)\right|_{h=1}
\end{aligned}
$$

From the product rule (14) it follows that

$$
\begin{aligned}
\mu_{k \mid k-1}(B)= & \int_{\mathcal{X}} \sum_{x \in \varphi}[\left.\delta G_{\mathrm{c}}\left(h \mid x ; 1_{B}\right)\right|_{h=1} \prod_{\substack{\bar{x} \in \varphi \\
\bar{x} \neq x}} \underbrace{G_{\mathrm{c}}(1 \mid \bar{x})}_{=1}] \\
& \times P_{k-1}(\mathrm{~d} \varphi)+\left.\delta G_{\gamma}\left(h ; 1_{B}\right)\right|_{h=1}
\end{aligned}
$$

Using the product rule (14) on $G_{\mathrm{c}}(\cdot \mid x)=G_{\mathrm{s}}(\cdot \mid x) G_{\mathrm{b}}(\cdot \mid x)$ then yields

$$
\begin{aligned}
\mu_{k \mid k-1}(B)= & \int_{\mathcal{X}} \sum_{x \in \varphi}[\left.\delta G_{\mathrm{S}}\left(h \mid x ; 1_{B}\right)\right|_{h=1} \underbrace{G_{\mathrm{b}}(1 \mid x)}_{=1} \\
& +\left.\underbrace{G_{\mathrm{S}}(1 \mid x)}_{=1} \delta G_{\mathrm{b}}\left(h \mid x ; 1_{B}\right)\right|_{h=1}] \\
& \times P_{k-1}(\mathrm{~d} \varphi)+\left.\delta G_{\gamma}\left(h ; 1_{B}\right)\right|_{h=1}
\end{aligned}
$$

Using Eq. (20) we introduce the intensity $\mu_{\mathrm{s}}$ (resp. $\mu_{\mathrm{b}}, \mu_{\gamma}$ ) of the survival (resp. spawning, spontaneous birth) process and we obtain:

$$
\mu_{k \mid k-1}(B)=\int_{\mathcal{X}} \sum_{x \in \varphi}\left[\mu_{\mathrm{s}}(B \mid x)+\mu_{\mathrm{b}}(B \mid x)\right] P_{k-1}(\mathrm{~d} \varphi)+\mu_{\gamma}(B)
$$

Which becomes, using Campbell's theorem [37, p. 271]:

$$
\mu_{k \mid k-1}(B)=\int\left[\mu_{\mathrm{s}}(B \mid x)+\mu_{\mathrm{b}}(B \mid x)\right] \mu_{k-1}(\mathrm{~d} x)+\mu_{\gamma}(B) .
$$

Note that the validity of the expression of the predicted intensity above is not restricted to specific models for the prior process $\Phi_{k-1}$. As such, the construction of the predicted intensity is identical in the case of the PHD filter with spawning (see Mahler's original proof in [5]). Let us now focus on the explicit expression of the intensity measure $\mu_{\mathrm{s}}$. Since the survival process is assumed Bernoulli with parameter $p_{\mathrm{S}}(\cdot)$ and spatial distribution $f_{\mathrm{S}}(\cdot \mid \cdot)$, we can exploit Eq. (20) to retrieve the intensity $\mu_{\mathrm{s}}$ through the expression of the p.g.fl. $G_{\mathrm{s}}$ given by Eq. (24):

$$
\begin{aligned}
\mu_{\mathrm{s}}(B \mid \cdot) & =\left.\delta G_{\mathrm{s}}\left(h \mid \cdot ; 1_{B}\right)\right|_{h=1} \\
& =\left.\delta\left(1-p_{\mathrm{s}}(\cdot)+p_{\mathrm{s}}(\cdot) \int h(x) f_{\mathrm{s}}(\mathrm{d} x \mid \cdot) ; 1_{B}\right)\right|_{h=1} \\
& =p_{\mathrm{s}}(\cdot) f_{\mathrm{s}}(B \mid \cdot)
\end{aligned}
$$

3) Predicted Cardinality: Let us now focus on the expression of the predicted cardinality $\rho_{k \mid k-1}$. From Eq. (4) the cardinality distribution of an arbitrary point process can be retrieved through its Janossy measures; let us then compute the predicted $n^{\text {th }}$-order Janossy measure $J_{k \mid k-1}^{(n)}$ evaluated at the neighborhood of a collection of $n$ arbitrary points $y_{1}, \ldots, y_{n}$. Using Eq. (19) yields

$$
\begin{aligned}
& J_{k \mid k-1}^{(n)}\left(\mathrm{d}\left(y_{1}, \ldots, y_{n}\right)\right) \\
& \quad=\left.\delta^{n} G_{k \mid k-1}\left(h ; 1_{\mathrm{d} y_{1}}, \ldots, 1_{\mathrm{d} y_{n}}\right)\right|_{h=0} \\
& \quad=\left.\delta^{n}\left(G_{k-1}\left(G_{\mathrm{c}}(h \mid \cdot)\right) G_{\gamma}(h) ; 1_{\mathrm{d} y_{1}}, \ldots, 1_{\mathrm{d} y_{n}}\right)\right|_{h=0}
\end{aligned}
$$


Applying the product rule (14) then gives

$$
\begin{aligned}
J_{k \mid k-1}^{(n)}( & \left.\mathrm{d}\left(y_{1}, \ldots, y_{n}\right)\right) \\
= & \left.\sum_{\tau \subseteq\{1, \ldots, n\}} \delta^{|\tau|}\left(G_{k-1}\left(G_{\mathrm{c}}(h \mid \cdot)\right) ;\left(1_{\mathrm{d} y_{i}}\right)_{i \in \tau}\right)\right|_{h=0} \\
& \times\left.\delta^{n-|\tau|} G_{\gamma}\left(h ;\left(1_{\mathrm{d} y_{i}}\right)_{i \in \tau^{c}}\right)\right|_{h=0}
\end{aligned}
$$

Using Eq. (19) on the instantaneous birth process then gives

$$
\begin{aligned}
& J_{k \mid k-1}^{(n)}\left(\mathrm{d}\left(y_{1}, \ldots, y_{n}\right)\right) \\
& =\sum_{\tau \subseteq\{1, \ldots, n\}} C_{|\tau|}\left(\mathrm{d}\left(y_{i}\right)_{i \in \tau}\right) J_{\gamma}^{(n-|\tau|)}\left(\mathrm{d}\left(y_{i}\right)_{i \in \tau^{c}}\right),
\end{aligned}
$$

where

$$
C_{|\tau|}\left(\mathrm{d}\left(y_{i}\right)_{i \in \tau}\right)=\left.\delta^{|\tau|}\left(G_{k-1}\left(G_{\mathrm{c}}(h \mid \cdot)\right) ;\left(1_{\mathrm{d} y_{i}}\right)_{i \in \tau}\right)\right|_{h=0},
$$

and where $J_{\gamma}^{(n-|\tau|)}$ is the $(n-|\tau|)^{\text {th }}$-order Janossy measure of the instantaneous birth process.

We shall now detail the expression of the quantity $C_{q}$, evaluated at the neighborhood of a collection of $q$ arbitrary points $z_{1}, \ldots, z_{q}$. Applying the general chain rule (12) yields

$$
\begin{aligned}
& C_{q}\left(\mathrm{~d}\left(z_{1}, \ldots, z_{q}\right)\right) \\
& =\left.\delta^{q}\left(G_{k-1}\left(G_{\mathrm{c}}(h \mid \cdot)\right) ; 1_{\mathrm{d} z_{1}}, \ldots, 1_{\mathrm{d} z_{q}}\right)\right|_{h=0} \\
& =\left.\sum_{\pi \in \Pi_{q}} \delta^{|\pi|} G_{k-1}\left(G_{\mathrm{c}}(h \mid \cdot) ;\left(\delta^{|\omega|} G_{\mathrm{c}}\left(h \mid \cdot ;\left(1_{\mathrm{d} z_{i}}\right)_{i \in \omega}\right)\right)_{\omega \in \pi}\right)\right|_{h=0} .
\end{aligned}
$$

Developing the predicted p.g.fl. $G_{k-1}$ through Janossy measures with Eq. (2) then gives

$$
\begin{aligned}
& C_{q}\left(\mathrm{~d}\left(z_{1}, \ldots, z_{q}\right)\right)=\sum_{\pi \in \Pi_{q}} \sum_{m \geq|\pi|} \frac{1}{(m-|\pi|) !} \\
& \quad \times\left.\int_{\mathbf{X}^{m}} \prod_{i=1}^{|\pi|} \delta^{\left|\omega_{i}\right|} G_{\mathrm{c}}\left(h \mid x_{i} ;\left(1_{\mathrm{d} z_{j}}\right)_{j \in \omega_{i}}\right)\right|_{h=0} \\
& \quad \times \prod_{i=|\pi|+1}^{m} G_{\mathrm{c}}\left(0 \mid x_{i}\right) J_{k-1}^{(m)}\left(\mathrm{d}\left(x_{1}, \ldots, x_{m}\right)\right) .
\end{aligned}
$$

Since the prior process is assumed i.i.d., we can substitute the expression given by Eq. (29) to the prior Janossy measures $J_{k-1}^{(m)}$ and obtain

$$
\begin{aligned}
& C_{q}\left(\mathrm{~d}\left(z_{1}, \ldots, z_{q}\right)\right) \\
& =\sum_{\pi \in \Pi_{q}} \sum_{m \geq|\pi|} \frac{m !}{(m-|\pi|) !} \rho(m) C_{\pi}\left(\mathrm{d}\left(z_{1}, \ldots, z_{q}\right)\right)
\end{aligned}
$$

where

$$
\begin{aligned}
C_{\pi}\left(\mathrm{d}\left(z_{1}, \ldots, z_{q}\right)\right)= & \left.\int \prod_{i=1}^{|\pi|} \delta^{\left|\omega_{i}\right|} G_{\mathrm{c}}\left(h \mid x_{i} ;\left(1_{\mathrm{d} z_{j}}\right){ }_{j \in \omega_{i}}\right)\right|_{h=0} \\
& \times \prod_{i=|\pi|+1}^{m} G_{\mathrm{c}}\left(0 \mid x_{i}\right) \prod_{i=1}^{m} s\left(\mathrm{~d} x_{i}\right) \\
= & \left(\int G_{\mathrm{c}}(0 \mid x) s(\mathrm{~d} x)\right)^{m-|\pi|} \\
& \times \prod_{\omega \in \pi}\left(\left.\int \delta^{|\omega|} G_{\mathrm{c}}\left(h \mid x ;\left(1_{\mathrm{d} z_{i}}\right)_{i \in \omega}\right)\right|_{h=0} s(\mathrm{~d} x)\right) .
\end{aligned}
$$

Recall from Eq. (61) that $G_{\mathrm{c}}(h \mid x)=G_{\mathrm{S}}(h \mid x) G_{\mathrm{b}}(h \mid x)$; using the product rule (14) on Eq. (68b) then yields

$$
\begin{aligned}
& C_{\pi}\left(\mathrm{d}\left(z_{1}, \ldots, z_{q}\right)\right)=\left(\int G_{\mathrm{s}}(0 \mid x) G_{\mathrm{b}}(0 \mid x) s(\mathrm{~d} x)\right)^{m-|\pi|} \\
& \quad \times \prod_{\omega \in \pi}\left(\left.\int \sum_{\nu \subseteq \omega} \delta^{|\nu|} G_{\mathrm{s}}\left(h \mid x ;\left(1_{\mathrm{d} z_{i}}\right)_{i \in \nu}\right)\right|_{h=0}\right. \\
& \left.\quad \times\left.\delta^{|\omega|-|\nu|} G_{\mathrm{b}}\left(h \mid x ;\left(1_{\mathrm{d} z_{i}}\right)_{i \in \omega \backslash \nu}\right)\right|_{h=0} s(\mathrm{~d} x)\right)
\end{aligned}
$$

Now, from the derivation shown in Eq. (64), we see that:

$$
\left.\delta^{|\nu|} G_{\mathrm{s}}\left(h \mid x ;\left(1_{\mathrm{d} z_{i}}\right){ }_{i \in \nu}\right)\right|_{h=0}= \begin{cases}1-p_{\mathrm{s}}(x), & \nu=\emptyset \\ p_{\mathrm{s}}(x) f_{\mathrm{s}}\left(\mathrm{d} z_{i} \mid x\right), & \nu=\{i\} \\ 0, & |\nu|>1\end{cases}
$$

Therefore, Eq. (69) simplifies as follows:

$$
\begin{aligned}
& C_{\pi}\left(\mathrm{d}\left(z_{1}, \ldots, z_{q}\right)\right)=\left(\int \bar{p}_{\mathrm{s}}(x) G_{\mathrm{b}}(0 \mid x) s(\mathrm{~d} x)\right)^{m-|\pi|} \\
& \times \prod_{\omega \in \pi}\left(\left.\int \bar{p}_{\mathrm{s}}(x) \delta^{|\omega|} G_{\mathrm{b}}\left(h \mid x ;\left(1_{\mathrm{d} z_{i}}\right)_{i \in \omega}\right)\right|_{h=0} s(\mathrm{~d} x)\right. \\
& \left.+\left.\int \sum_{i \in \omega} p_{\mathrm{s}}(x) f_{\mathrm{s}}\left(\mathrm{d} z_{i} \mid x\right) \delta^{|\omega|-1} G_{\mathrm{b}}\left(h \mid x ;\left(1_{\mathrm{d} z_{j}}\right)_{j \in \omega \backslash i}\right)\right|_{h=0} s(\mathrm{~d} x)\right),
\end{aligned}
$$

Which becomes, using Eq. (19):

$$
\begin{aligned}
& C_{\pi}\left(\mathrm{d}\left(z_{1}, \ldots, z_{q}\right)\right)=\left(\int \bar{p}_{\mathrm{s}}(x) J_{\mathrm{b}}^{(0)}(x) s(\mathrm{~d} x)\right)^{m-|\pi|} \\
& \times \prod_{\omega \in \pi}\left(\int \bar{p}_{\mathrm{s}}(x) J_{\mathrm{b}}^{(|\omega|)}\left(\mathrm{d}\left(z_{i}\right)_{i \in \omega} \mid x\right) s(\mathrm{~d} x)\right. \\
& \left.+\int \sum_{i \in \omega} p_{\mathrm{s}}(x) f_{\mathrm{s}}\left(\mathrm{d} z_{i} \mid x\right) J_{\mathrm{b}}^{(|\omega|-1)}\left(\mathrm{d}\left(z_{j}\right)_{j \in \omega \backslash i} \mid x\right) s(\mathrm{~d} x)\right),
\end{aligned}
$$


where $J_{\mathrm{b}}^{(|\omega|)}$ is the $|\omega|^{\text {th }}$-order Janossy measure of the spawning process. Exploiting Eq. (4), it follows from Eq. (71b) that

$$
\begin{aligned}
\int_{\mathbf{X}^{q}} C_{\pi}\left(\mathrm{d}\left(z_{1}, \ldots, z_{q}\right)\right)= & \left(\int \bar{p}_{\mathrm{s}}(x) \rho_{\mathrm{b}}(0 \mid x) s(\mathrm{~d} x)\right)^{m-|\pi|} \\
& \times \prod_{\omega \in \pi}|\omega| ! \int\left[\bar{p}_{\mathrm{s}}(x) \rho_{\mathrm{b}}(|\omega| \mid x)\right. \\
& \left.+p_{\mathrm{s}}(x) \rho_{\mathrm{b}}(|\omega|-1 \mid x)\right] s(\mathrm{~d} x)(72 \mathrm{a}) \\
= & b_{0}^{m-|\pi|} \prod_{\omega \in \pi} b_{|\omega|}, \quad \text { (72b) }
\end{aligned}
$$

where the coefficients $b_{i}$ are defined by

$$
b_{i}= \begin{cases}\int \bar{p}_{\mathrm{s}}(x) \rho_{\mathrm{b}}(0 \mid x) s(\mathrm{~d} x), & i=0, \\ i ! \int\left[\bar{p}_{\mathrm{s}}(x) \rho_{\mathrm{b}}(i \mid x)+p_{\mathrm{s}}(x) \rho_{\mathrm{b}}(i-1 \mid x)\right] s(\mathrm{~d} x), & i>0 .\end{cases}
$$

Exploiting Eq. (72b), it follows from Eq. (67d) that

$$
\begin{aligned}
& \int_{\mathbf{X}^{q}} C_{q}\left(\mathrm{~d}\left(z_{1}, \ldots, z_{q}\right)\right) \\
& =\sum_{\pi \in \Pi_{q}} \sum_{m \geq|\pi|} \frac{m !}{(m-|\pi|) !} \rho(m) b_{0}^{m-|\pi|} \prod_{\omega \in \pi} b_{|\pi|} .
\end{aligned}
$$

We may finally retrieve the scalar $\rho_{k \mid k-1}(n)$ through Eq. (4):

$$
\begin{aligned}
\rho_{k \mid k-1}(n)= & \frac{1}{n !} \int_{\mathbf{X}^{n}} J_{k \mid k-1}^{(n)}\left(\mathrm{d}\left(y_{1}, \ldots, y_{n}\right)\right) \\
= & \sum_{\nu \subseteq\{1, \ldots, n\}} \frac{1}{(n-|\nu|) !} \int_{\mathbf{X}^{n-|\nu|}} J_{\gamma}^{(n-|\nu|)}\left(\mathrm{d}\left(y_{i}\right)_{i \in \nu^{c}}\right) \\
& \times \frac{(n-|\nu|) !}{n !} \int_{\mathbf{X}^{|\nu|}} C_{|\nu|}\left(\mathrm{d}\left(y_{i}\right)_{i \in \nu}\right)
\end{aligned}
$$$$
=\sum_{q=0}^{n}\left(\begin{array}{l}
n \\
q
\end{array}\right) \rho_{\gamma}(n-q) \frac{(n-q) !}{n !}
$$$$
\times \sum_{\pi \in \Pi_{q}} \sum_{m \geq|\pi|} \frac{m !}{(m-|\pi|) !} \rho(m) b_{0}^{m-|\pi|} \prod_{\omega \in \pi} b_{\mid \omega}
$$$$
=\sum_{q=0}^{n} \rho_{\gamma}(n-q) \sum_{\pi \in \Pi_{q}} \sum_{m \geq|\pi|} \frac{m !}{q !(m-|\pi|) !}
$$$$
\times \rho(m) b_{0}^{m-|\pi|} \prod_{\omega \in \pi} b_{|\omega|} .
$$

Using the definition of the Bell polynomial (32) then yields the desired result.

\section{B. Proof of Corollary 1}

For the sake of simplicity, the time subscripts will be omitted throughout the proof when there is no ambiguity.

1) Predicted Intensity: Let us first focus on the explicit expression of the intensity measure $\mu_{\mathrm{b}}$ of the spawning process in
Eq. (30), depending on the modeling choices for the spawning process.

a) Bernoulli process with parameter $p_{\mathrm{b}}(\cdot)$ and spatial distribution $s_{\mathrm{b}}(\cdot \cdot \cdot)$ : Using the same construction as in Eq. (64) we have immediately

$$
\mu_{\mathrm{b}}(B \mid \cdot)=p_{\mathrm{b}}(\cdot) s_{\mathrm{b}}(B \mid \cdot) .
$$

b) zero-inflated Poisson process with parameter $p_{\mathrm{b}}(\cdot)$, rate $\lambda_{\mathrm{b}}(\cdot)$ and spatial distribution $s_{\mathrm{b}}(\cdot \mid \cdot)$ : Exploiting Eq. (28) yields

$$
\begin{aligned}
& \mu_{\mathrm{b}}(B \mid \cdot) \\
& =\left.\delta G_{\mathrm{b}}\left(h \mid \cdot ; 1_{B}\right)\right|_{h=1} \\
& =\left.\delta\left(\bar{p}_{\mathrm{b}}(\cdot)+p_{\mathrm{b}}(\cdot) \exp \left[\lambda_{\mathrm{b}}(\cdot)\left(\int h(x) s_{\mathrm{b}}(\mathrm{d} x \mid \cdot)-1\right)\right] ; 1_{B}\right)\right|_{h=1} \\
& =\left.p_{\mathrm{b}}(\cdot) \lambda_{\mathrm{b}}(\cdot) \delta\left(\int h(x) s_{\mathrm{b}}(\mathrm{d} x \mid \cdot)-1 ; 1_{B}\right)\right|_{h=1} \\
& \times \exp \underbrace{\left[\lambda_{\mathrm{b}}(\cdot)\left(\int s_{\mathrm{b}}(\mathrm{d} x \mid \cdot)-1\right)\right]}_{=0} \\
& =p_{\mathrm{b}}(\cdot) \lambda_{\mathrm{b}}(\cdot) s_{\mathrm{b}}(B \mid \cdot) .
\end{aligned}
$$

2) Predicted Cardinality: Let us now detail the expression of the coefficients $b_{i}$ of the Bello polynomial in Eq. (33), depending on the modeling choices for the spawning process.

a) Bernoulli process with parameter $p_{\mathrm{s}}(\cdot)$ and spatial distribution $f_{\mathrm{s}}(\cdot \mid \cdot)$ : From the description of the Bernoulli process (23) it follows that

$$
\rho_{\mathrm{b}}(n \mid x)= \begin{cases}\bar{p}_{\mathrm{b}}(x), & n=0, \\ p_{\mathrm{b}}(x), & n=1, \\ 0, & \text { otherwise. }\end{cases}
$$

Thus, the coefficients $b_{i}$ in Eq. (33) become

$$
b_{i}= \begin{cases}\int \bar{p}_{\mathrm{s}}(x) \bar{p}_{\mathrm{b}}(x) s(\mathrm{~d} x), & i=0, \\ \int\left[p_{\mathrm{s}}(x) \bar{p}_{\mathrm{b}}(x)+\bar{p}_{\mathrm{s}}(x) p_{\mathrm{b}}(x)\right] s(\mathrm{~d} x), & i=1, \\ 2 \int p_{\mathrm{s}}(x) p_{\mathrm{b}}(x) s(\mathrm{~d} x), & i=2, \\ 0, & i>2 .\end{cases}
$$

b) zero-inflated Poisson process with parameter $p_{\mathrm{b}}(\cdot)$, rate $\lambda_{\mathrm{b}}(\cdot)$, and spatial distribution $s_{\mathrm{b}}(\cdot \cdot \cdot)$ : From the description of the zero-inflated Poisson process (27) it follows that

$$
\rho_{\mathrm{b}}(n \mid x)= \begin{cases}\bar{p}_{\mathrm{b}}(x)+p_{\mathrm{b}}(x) e^{-\lambda_{\mathrm{b}}(x)}, & n=0, \\ p_{\mathrm{b}}(x) e^{-\lambda_{\mathrm{b}}(x) \frac{\lambda_{\mathrm{b}}(x)^{n}}{n !},} & \text { otherwise. }\end{cases}
$$

Thus, the coefficients $b_{i}$ in Eq. (33) become

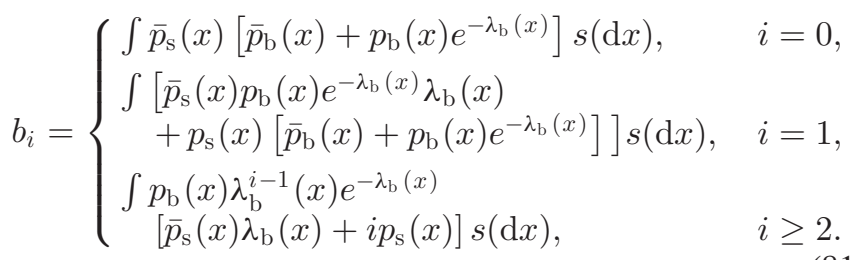




\section{Comparison of Expressions [15, (A.15)] and Eq. (31)}

The construction of the predicted cardinality in [15] relies on the derivation of p.g.fs, describing the cardinality distribution of specific processes, through the Faà di Bruno's general chain formula for usual derivatives (more information on p.g.fs in the context of multi-object filtering can be found in [3], [5]). The connection between the two expressions can be established through the version of Faà di Bruno's formula involving partial Bell polynomials [13, p.420], i.e.,

$$
\begin{aligned}
& \frac{\mathrm{d}^{n}}{\mathrm{~d} \mathbf{x}^{n}} F(G(\mathbf{x})) \\
& \quad=\sum_{k=0}^{n} F^{(k)}(G(\mathbf{x})) B_{n, k}\left(G^{(1)}(\mathbf{x}), \ldots, G^{(n)}(\mathbf{x})\right),
\end{aligned}
$$

where $F, G$ denote some suitable functions. Substituting Eq. (82) in [15, (A.9)] yields

$$
\begin{aligned}
& \frac{1}{q !} \frac{\mathrm{d}^{q}}{\mathrm{~d} \mathbf{x}^{q}} G_{k-1}(g(\mathbf{x})) \\
& \quad=\frac{1}{q !} \sum_{j=0}^{q} G_{k-1}^{(j)}(g(\mathbf{x})) B_{q, j}\left(g^{(1)}(\mathbf{x}), \ldots, g^{(q)}(\mathbf{x})\right),
\end{aligned}
$$

where

$$
\begin{aligned}
g(\mathbf{x})= & \int\left[\bar{p}_{\mathrm{s}, k}(x)+p_{\mathrm{s}, k}(x) \mathbf{x}\right] G_{\mathrm{b}, k}(\mathbf{x} \mid x) s_{k-1}(\mathrm{~d} x) \\
g^{(i)}(\mathbf{x})= & \int\left[\bar{p}_{\mathrm{s}, k}(x) G_{\mathrm{b}, k}^{(i)}(\mathbf{x} \mid x)+i p_{\mathrm{s}, k}(x)\right. \\
& \left.\times G_{\mathrm{b}, k}^{(i-1)}(\mathbf{x} \mid x)\right] s_{k-1}(\mathrm{~d} x)
\end{aligned}
$$

where $i \geq 1$, and where $G_{k-1}$ (resp. $G_{\mathrm{b}, k}$ ) denotes the p.g.f of the prior (resp. spawning) process. (Note that, for the sake of simplicity, we use the same notation for the p.g.f and p.g.fl. of a process, though the quantities are different in nature.) Following $[15,(\mathrm{~A} .7)]$, we then have

$$
\begin{aligned}
& \left.\frac{1}{n !} \frac{\mathrm{d}^{n}}{\mathrm{~d} \mathbf{x}^{n}} G_{k \mid k-1}(\mathbf{x})\right|_{\mathbf{x}=0} \\
& =\sum_{q=0}^{n} \rho_{\gamma, k}(n-q) \frac{1}{q !} \sum_{j=0}^{q} G_{k-1}^{(j)}(g(0)) \\
& \quad \times B_{q, j}\left(g^{(1)}(0), \ldots, g^{(n)}(0)\right),
\end{aligned}
$$

where $G_{k \mid k-1}$ denotes the p.g.f of the predicted process. Using basic calculus properties on p.g.fs, we have

$$
\begin{aligned}
G_{k-1}^{(j)}(g(0)) & =\sum_{m \geq j} \frac{m !}{(m-j) !} \rho_{k-1}(m)(g(0))^{m-j} \\
& =\sum_{m \geq j} \frac{m !}{(m-j) !} \rho_{k-1}(m) b_{0}^{m-j}
\end{aligned}
$$

Also, from Eq. (85) we have

$$
\begin{aligned}
g^{(i)}(0)= & \int\left[\bar{p}_{\mathrm{s}, k}(x) G_{\mathrm{b}, k}^{(i)}(0 \mid x)+i p_{\mathrm{s}, k}(x) G_{\mathrm{b}, k}^{(i-1)}(0 \mid x)\right] \\
& \times s_{k-1}(\mathrm{~d} x) \\
= & i ! \int\left[\bar{p}_{\mathrm{s}, k}(x) \rho_{\mathrm{b}, k}(i \mid x)+i p_{\mathrm{s}, k}(x) \rho_{\mathrm{b}, k}(i-1 \mid x)\right] \\
& \times s_{k-1}(\mathrm{~d} x) \\
= & b_{i},
\end{aligned}
$$

where $i \geq 1$. Substituting Eq. (87b) and Eq. (88c) into Eq. (86), the predicted cardinality [15, (A.15)] then takes the form (31).

\section{ACKNOWLEDGMENT}

Daniel Clark wishes to thank Professor Penina Axelrad in the Department of Aerospace Engineering Sciences at the University of Colorado at Boulder for supporting his Visiting Professor position through the faculty-in-residence summer term (FIRST) programme at the University of Colorado Boulder in summer 2014. The authors would also like to thank Nicola Baresi and In-Kwan Park of the University of Colorado at Boulder and Illán Amor of Universidad de Oviedo, Asturias Spain for their conversations and ideas early on for this work during the FIRST programme.

\section{REFERENCES}

[1] T. E. Fortmann, Y. Bar-Shalom, and M. Scheffe, "Sonar tracking of multiple targets using joint probabilistic data association," IEEE J. Ocean. Eng., vol. 8, no. 3, pp. 173-184, Jul.1983.

[2] D. Reid, "An algorithm for tracking multiple targets," IEEE Trans. Autom. Control, vol. 24, no. 6, pp. 843-854, Dec. 1979.

[3] R. P. S. Mahler, Statistical Multisource-Multitarget Information Fusion. Norwood, MA: Artech House, 2007.

[4] R. P. S. Mahle, Advances in Statistical Multisource-Multitarget Information Fusion. Norwood, MA, USA: Artech House, 2014.

[5] R. P. S. Mahle, "Multitarget bayes filtering via first-order multitarget moments," IEEE Trans. Aerosp. Electron. Syst., vol. 39, no. 4, pp. 1152-1178, Oct. 2003.

[6] R. P. S. Mahle, "PHD filters of higher order in target number," IEEE Trans. Aerosp. Electron. Syst., vol. 43, no. 4, pp. 1523-1543, Oct. 2007.

[7] M. Swartwout, "A brief history of rideshares (and attack of the CubeSats)," in Proc. IEEE Aerosp. Conf, Mar. 2011, pp. 1-15.

[8] M. Swartwout, "A statistical survey of rideshares (and attack of the CubeSats, part deux)," in Proc. IEEE Aerosp. Conf., Mar. 2012, pp. 1-7.

[9] L. Anselmo and C. Pardini, "Analysis of the consequences in low Earth orbit of the collision between Cosmos 2251 and Iridium 33," in Proc. 21st Int. Symp. Space Flight Dyn., Sep. 2008, vol. 294.

[10] N. L. Johnson, E. Stansbery, J.-C. Liou, M. Horstman, C. Stokely, and D. Whitlock, "The characteristics and consequences of the break-up of the Fengyun-1C spacecraft," Acta Astronaut., vol. 63, no. 1, pp. 128-135, 2008.

[11] B. A. Jones, D. S. Bryant, B.-T. Vo, and B.-N. Vo, "Challenges of multi-target tracking for space situational awareness," in Proc. 18th Int. Conf. Inf. Fusion, Jul. 2015, pp. 1278-1285.

[12] B. A. Jones, S. Gehly, and P. Axelrad, "Measurement-based birth model for a space object cardinalized probability hypothesis density filter," in Proc. AIAA/AAS Astrodyn. Spec. Conf., vol. 4311, Aug. 2014, Art. no. 2014.

[13] C. A. Charalambides, Enumerative Combinatorics. Boca Raton, FL, USA: CRC Press, 2002.

[14] M. Lundgren, L. Svensson, and L. Hammarstrand, "A CPHD filter for tracking with spawning models," IEEE J. Sel. Topics Signal Process., vol. 7, no. 3, pp. 496-507, Jun. 2013. 
[15] M. Lundgren, L. Svensson, and L. Hammarstrand, "A CPHD filter for tracking with spawning models-Including a FISST based derivation," Chalmers Univ. of Technology, Gothenburg, Sweden, Tech. Rep. no. R006/2012, 2012

[16] P. Jing, J. Zou, Y. Duan, S. Xu, and Z. Chen, "Generalized CPHD filter modeling spawning targets," Signal Process., vol. 128, pp. 48-56, 2016.

[17] D. S. Bryant, E. D. Delande, S. Gehly, J. Houssineau, D. E. Clark, and B. A. Jones, "Spawning models for the CPHD filter," 2015, arXiv: $1507.00033 \mathrm{v} 1$.

[18] D. Stoyan, W. S. Kendall, and J. Mecke, Stochastic Geometry and Its Applications, 2nd ed. Hoboken, NJ, USA: Wiley, 1995.

[19] B.-N. Vo, S. Singh, and A. Doucet, "Sequential Monte Carlo methods for multi-target filtering with random finite sets," IEEE Trans. Aerosp. Electron. Syst., vol. 41, no. 4, pp. 1224-1245, Oct. 2005.

[20] D. Vere-Jones and D. J. Daley, An Introduction to the Theory of Point Processes, 2nd ed. (ser. Statistical Theory and Methods), D. Vere-Jones and D. J. Daley, Eds. New York, NY, USA: Springer, 2003, vol. 1.

[21] E. D. Delande, M. Uney, J. Houssineau, and D. E. Clark, "Regional variance for multi-object filtering," IEEE Trans. Signal Process., vol. 62, no. 13 , pp. $3415-3428$, Jul. 2014

[22] S. K. Srinivasan and A. Vijayakumar, Point Processes and Product Densities. Oxford U.K.: Alpha Science International Ltd., 2003.

[23] J. E. Moyal, "The general theory of stochastic population processes," Acto Math., vol. 108, no. 1, pp. 1-31, 1962. families," J. Anthropological Inst. Great Britain, vol. 4, pp. 138-144, 1875.

[25] P. Bernhard, "Chain differentials with an application to the mathematical fear operator," Nonlinear Anal., vol. 62, no. 7, pp. 1225-1233, 2005.

[26] D. E. Clark and J. Houssineau, "Faà di Bruno's formula and spatial cluster modelling," Spatial Stat., vol. 6, pp. 109-117, 2013.

[27] D. E. Clark and J. Houssineau, "Faà di Bruno's formula for chain differentials," arXiv:1310.2833, 2013.

[28] F. Faà di Bruno, "Sullo Sviluppo delle Funzioni," Annali di Scienze Matem-

[29] J. Houssineau and D. E. Clark, "Bayesian estimation of multi-object systems with independently identically distributed correlations," in Proc. IEEE Stat. Signal Process. Workshop, Jun. 2014, pp. 228-231.

[30] D. E. Clark, J. Houssineau, and E. Delande, "A few calculus rules for chain differentials," arXiv:1506.08626, 2015.

[31] S. K. Srinivasan, Stochastic Point Processes and Their Applications, ser. Griffin's Statistical Monographs and Courses. London, U.K., Griffin, 1973.

[32] B.-T. Vo, B.-N. Vo, and A. Cantoni, "Analytic implementations of the cardinalized probability hypothesis density filter" IEEE Trans. Signal Process., vol. 55, no. 7, pp. 3553-3567, Jul. 2007.

33] D. Lambert, "Zero-inflated poisson regression, with an application to defects in manufacturing," Technometrics, vol. 34, no. 1, pp. 1-14, 1992.

[34] B.-N. Vo and W.-K. Ma, "The Gaussian Mixture Probability Hypothesis Density Filter," IEEE Trans. Signal Process., vol. 54, no. 11, pp. 4091-4104, Nov. 2006.

[35] D. Schuhmacher, B.-N. Vo, and B.-T. Vo, "A consistent metric for performance evaluation of multi-object filters," IEEE Trans. Signal Process. vol. 56, no. 8, pp. 3447-3457, Aug. 2008.

[36] A. L. Gibbs and F. E. Su, "On choosing and bounding probability metrics," Int. Stat. Rev., vol. 70, no. 3, pp. 419-435, 2002.

[37] D. Vere-Jones and D. J. Daley, An Introduction to the Theory of Point Processes, 2nd ed. (ser. Statistical Theory and Methods), D. Vere-Jones and D. J. Daley, Eds. New York, USA: Springer, 2008, vol. 2.
[24] H. W. Watson and F. Galton, "On the probability of the extinction of atiche e Fisiche, vol. 6, pp. 479-480, 1855 .

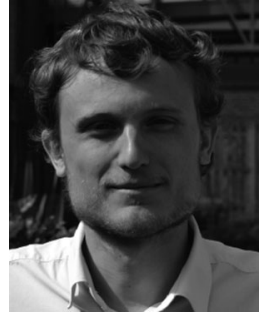

Emmanuel D. Delande received an Eng. degree from the grande école Ecole Centrale de Lille, Lille, and a M.Sc. degree in automatic control and signal processing from the University of Science \& Technology, Lille, both in 2008. He was awarded his Ph.D. degree from the Ecole Centrale de Lille in 2012. He is currently with the School of Engineering and Physical Sciences at Heriot-Watt University in Edinburgh, where he was awarded a Postdoctoral position in the EPSRC-DSTL grant Signal Processing 4 the Networked Battlespace in 2013. His research interests are in the design and the implementation of multiobject filtering solutions for multiple target tracking and sensor management problems.

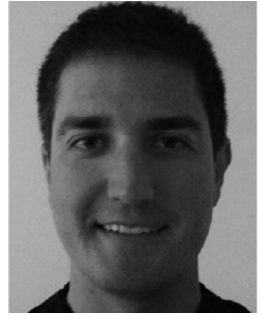

Steve Gehly was born in Boston, MA. He received his B.S. and M.S. degrees in aerospace engineering from the University of Southern California. He is currently working towards his $\mathrm{Ph} . \mathrm{D}$. degree in aerospace engineering at the University of Colorado. His research is focused on multi-target estimation and sensor allocation for tracking space objects.

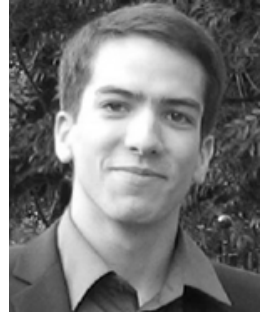

Jérémie Houssineau is a Research Fellow in the department of Statistics and Applied Probability at the University of Singapore. He received an Eng. degree in mathematical and mechanical modelling from MATMECA, Bordeaux, and a M.Sc. degree in mathematical modelling and statistics from the University of Bordeaux, both in 2009. From 2009 to 2011, he was a Research Engineer with DCNS, Toulon, involved in the design and assessment of statistical multi-sensor multi-object estimation algorithms. He was awarded a Ph.D. in Statistical Signal Processing in 2015 from Heriot-Watt University, Edinburgh.

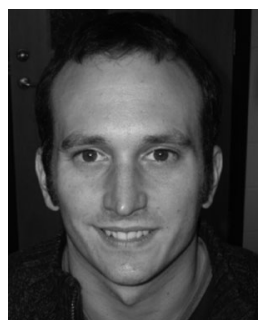

Daniel E. Clark is an Associate Professor in the School of Engineering and Physical Sciences at Heriot-Watt University. His research interests are in the development of the theory and applications of multi-object estimation algorithms for sensor fusion problems. He has led a range of projects spanning theoretical algorithm development to practical deployment. He was awarded his Ph.D. in 2006 from Heriot-Watt University.

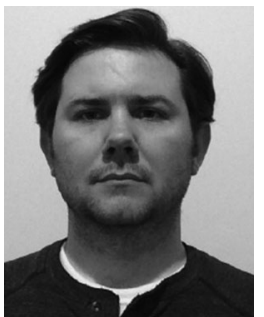

Daniel S. Bryant received a B.S. degree in mechanical engineering from the University of Texas at Arlington in 2011 and an M.S. degree in aerospace engineering sciences focusing in astrodynamics and satellite navigation from the University of Colorado at Boulder in 2013. He is currently working toward his $\mathrm{Ph} . \mathrm{D}$. degree in the aerospace engineering sciences program at the University of Colorado at Boulder and is supported by the Science, Mathematics, and Research for Transformation Scholarship Program. His research interests include space situational awareness and the development of multi-object tracking algorithms.

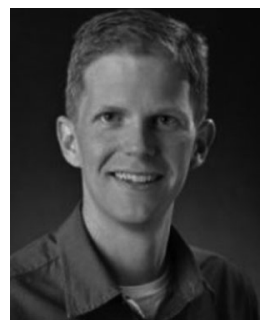

Brandon A. Jones was awarded his Ph.D. in Aerospace Engineering Sciences in 2010, where he was also a research assistant professor until $2015 . \mathrm{He}$ is currently an assistant professor at The University of Texas at Austin in the Department of Aerospace Engineering and Engineering Mechanics. His research interests include space situational awareness, multitarget tracking, computational methods for orbit state and uncertainty propagation, and satellite navigation. 